\title{
Turbulence Amplitude Amplification in an Externally Forced, Subsonic Turbulent Boundary Layer
}

\author{
Piyush Ranade* \\ University of Notre Dame, Notre Dame, Indiana 46556 \\ Subrahmanyam Duvvuri⿱亠 and Beverley McKeon \\ California Institute of Technology, Pasadena, California 91125 \\ and \\ Stanislav Gordeyev, $\underline{\S}$ Kenneth Christensen, $\mathbb{I}$ and Eric J. Jumper*** \\ University of Notre Dame, Notre Dame, Indiana 46556
}

\section{DOI: $10.2514 / 1 . J 057871$}

\begin{abstract}
Experimental studies of the changes in turbulence characteristics inside a boundary layer due to external forcing were performed using hot-wire anemometry. The forcing was created by a periodically forced shear layer that was external to a compressible subsonic turbulent boundary layer. The convecting coherent structures in the shear layer create a concomitant unsteady pressure and velocity field and provide an external disturbance for the turbulent boundary layer on the wall of the tunnel close to the forced shear layer. Both the pressure and velocity fluctuations inside the boundary layer were simultaneously measured along with the forcing signal, and a phase-locked analysis was performed. Regions of amplified turbulence inside the boundary layer were observed. Near the wall, the region of amplified turbulence was slightly upstream or lagging of the external forcing and away from the wall it was downstream or leading the forcing signal. Analysis of the convective speeds in the region of amplified turbulence supported the existence of the critical layer inside the wake region of the boundary layer, and the critical layer is believed to be responsible for the amplified levels of the turbulence in the wake region. Various modulation and amplification correlation coefficients were computed and analyzed, and the results also indicated the presence of the critical layer. Examination of the phase-locked turbulence revealed similarities between the turbulence amplitude amplification results due to these externally forced experiments and modulation response of an internally forced, subsonic boundary layer in the literature.
\end{abstract}

\begin{tabular}{lll} 
& & \multicolumn{1}{c}{ Nomenclature } \\
$A, B$ & $=$ & King's law coefficients \\
$a$ & $=$ & speed of sound \\
$C$ & $=$ & constant in log-law equation \\
$f_{\text {force }}$ & $=$ & forcing frequency \\
$f_{s}$ & $=$ & sampling frequency \\
$E$ & $=$ & voltage \\
$H$ & $=$ & shape factor, $\delta^{*} / \theta$ \\
$l$ & $=$ & hot-wire length \\
$M$ & $=$ & Mach number \\
$m$ & $=$ & exponent in King's law
\end{tabular}

Presented as Paper 2016-1120 at the 54th AIAA Aerospace Sciences Meeting, San Diego, CA, 4-8 January 2016; received 31 August 2018; revision received 26 April 2019; accepted for publication 21 May 2019; published online 25 June 2019. Copyright (C) 2019 by Ranade, Duvvuri, McKeon, Gordeyev, Christensen, and Jumper. Published by the American Institute of Aeronautics and Astronautics, Inc., with permission. All requests for copying and permission to reprint should be submitted to CCC at www. copyright.com; employ the eISSN 1533-385X to initiate your request. See also AIAA Rights and Permissions www.aiaa.org/randp.

*Graduate Student, Hessert Laboratory for Aerospace Research, Department of Mechanical and Aerospace Engineering, University of Notre Dame, Notre Dame, Indiana 46556; currently R\&D Scientist, Honeywell Aerospace, Plymouth, Minnesota 55441. Student Member AIAA.

${ }^{\dagger}$ Assistant Professor, Department of Aerospace Engineering, Indian Institute of Science, Bengaluru 560 012, India.

־Theodore von Karman Professor of Aeronautics, Graduate Aerospace Laboratories. Associate Fellow AIAA.

${ }^{\S}$ Associate Professor, Hessert Laboratory for Aerospace Research, Department of Mechanical and Aerospace Engineering. Associate Fellow AIAA.

†Viola D. Hank Professor, Hessert Laboratory for Aerospace Research, Department of Mechanical and Aerospace Engineering; also Department of Civil \& Environmental Engineering \& Earth Sciences. Associate Fellow AIAA.

**Roth-Gibson Professor, Hessert Laboratory for Aerospace Research, Department of Mechanical and Aerospace Engineering. Fellow AIAA.

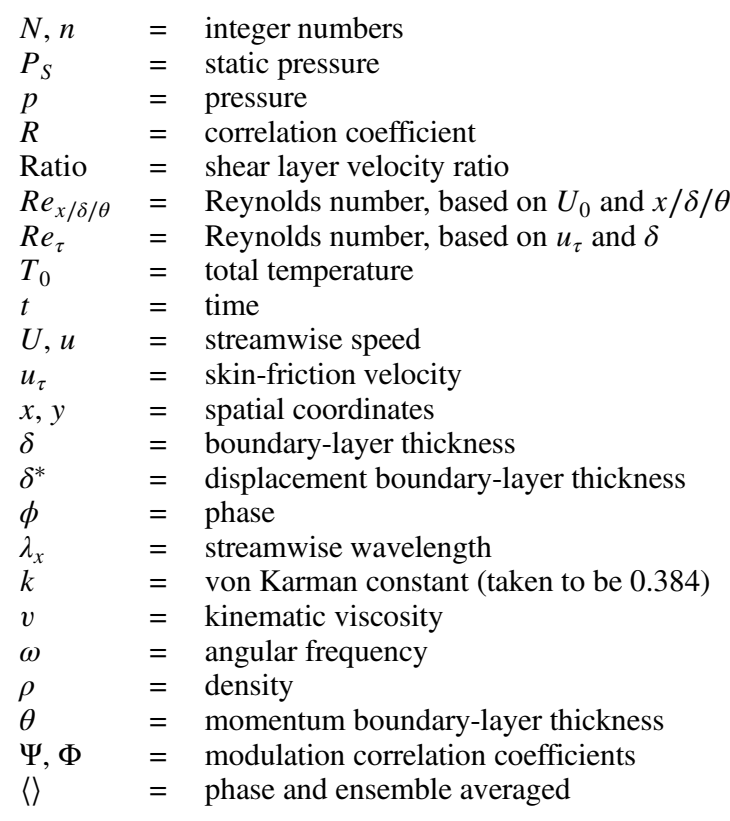

Superscripts

$\begin{array}{ll}\sim & =\text { phase-locked averaged } \\ - & =\text { time-averaged } \\ , & =\text { mean-removed fluctuations } \\ + & =\text { inner units }\end{array}$

Subscripts

$C=$ convective

$L \quad=$ large-scale

rms $=$ root-mean-square

$S=$ small-scale 
$\begin{array}{lll}0 & = & \text { high-speed } \\ 1 & = & \text { low-speed }\end{array}$

\section{Introduction}

$\mathbf{T}$ HE phenomenon of turbulence modulation within a turbulent boundary layer due to a nonlinear interaction between different structures in the boundary layer has been studied since the late $1960 \mathrm{~s}$ [1,2]. In 1984, Bandyopadhyay and Hussain [3] used a method, introduced in $[2,4]$ to decompose the time series of the fluctuating velocity signal into its large-scale and small-scale components using a Butterworth digital filter. A correlation coefficient was then used to correlate the low-frequency (large-scales) component of the fluctuating velocity signal with the envelope of the high-frequency (small-scales) component. Their experiments revealed a relationship between the large- and small-scales in a phenomenon they termed "inner-outer interaction." Their experiments revealed a positive correlation close to the wall, intermediate correlation in the log region, and a negative correlation (phase reversal) in the wake region and farther away from the wall. Hutchins and Marusic [5] expanded on this idea and applied it to flows of high Reynolds numbers. By plotting the energy spectra of the unfiltered signal, they saw two clear peaks. One peak was in the inner portion of the boundary layer, corresponding to a structure size of 1000 inner units in the streamwise direction. The "inner" peak was the energetic signature of the viscous-scaled near-wall structures commonly found throughout the literature. The second peak was in the outer boundary layer, with the structure size of several boundary-layer thicknesses. They also applied a spectral filter at a wavelength equal to the boundary-layer thickness to separate the large- and small-scales in similar fashion as in [3] and noticed that the large-scale fluctuating velocity signal seemed to modulate the small-scale signal, as near the wall negative large-scale velocity excursions correspond to reduced amplitude of the small-scale fluctuations. Farther away from the wall than the location of the outer peak, the trend reversed and the negative largescale velocity excursion corresponded to increased small-scale activity. They stated that the "low-wave number motions (associated with the log-region structure) influence the near-wall u-fluctuations in a manner akin to pure amplitude modulation," introducing the idea that the envelope of the large-scales "carried" the small-scales, such that even upon removal of the large-scale component, the modulation was still visible in the small-scales.

Mathis et al. [6] built upon the previous findings in [3] and used the Hilbert transformation to formally quantify the modulation of smaller scales by larger scales. The Hilbert transformation was used to obtain the envelope of fluctuating velocity signals. The envelope of the small-scale component was further filtered at the same cutoff criteria as in [5] to remove the effect of small-scale variation in the carrier signal due to factors other than the large-scale signal, leaving only the component of the small-scale envelope influenced by the largescales. The algorithm used in [6] is fundamentally similar to []ㅡ, but instead correlates the large-scale component with the low-passfiltered envelope of the small-scale component. Using two hotwires to obtain the two fluctuating signals at the inner and the outer locations, the authors in [6] calculated the correlation coefficient between the filtered envelope of the small-scale at the inner location and the large-scale component at the outer location and found that a strong positive correlation existed between the large- and smallscales near the wall, a region of zero correlation coefficient existed in the log layer, and a strong negative correlation existed in the outer boundary layer. All of it gave quantifiable evidence of the perceived notion of large-scale motions in the outer boundary layer leaving a footprint on and modulating the small-scale motions near the wall. Mathis et al. [7] used the same definition of the correlation coefficient to show that apart from some slight deviations in the outer region, the magnitude and shape of the correlation coefficient is very similar for flows in pipes and channels, and compares extremely well with [6]. Using these results, a semi-empirical statistically representative inner-outer model was developed in [8], which was demonstrated to correctly predict velocity spectra and different moments over a large range of Reynolds numbers. Finally, it is worth noting that
Townsend's attached-eddy hypothesis [9] laid the groundwork for linking the statistical and structural views of turbulence by finding why statistical arguments concerning the velocity profile and velocity fluctuations make physical sense.

To explain the experimentally observed modulation effect of the large-scale structures on near-wall turbulence, a quasi-steady theoretical model was developed in $[10,11]$, where it was assumed that the near-wall turbulence responds to large-scale motion in a quasi-steady fashion; that is, the slowly varying skin friction depends only on the low-pass filtered friction velocity. Using the Reynoldsnumber-independent representation of the skin friction and the velocity, scaled in wall units, the model was able to correctly predict both the modulation and the superposition effects, observed experimentally in [6] and to justify the empirical correlation between near-wall fluctuations and the large-scale structures, used to develop the predictive model for the inner-outer scale interaction [8].

It is also now generally accepted that very large-scale motion (VLSM) structures, defined as structures with streamwise extent of several boundary-layer thicknesses [12], are responsible for modulating the small-scales near the wall. It is believed that single hairpin vortices align within the areas of low streamwise momentum created between the legs of the superstructure, and thus the smallscale, high-frequency velocity signal naturally is "carried" within the footprint of the large-scale, low-frequency velocity signal. Hutchins and Marusic [5] had noted that the "outer" peak location in their streamwise energy spectra scaled with boundary-layer thickness and was most likely the energetic signature of the VLSMs. Jacobi and McKeon [13] used a cospectral technique to show that a wavenumber matching the VLSM was responsible for the strongest modulating influence of the large-scale signal.

Related to the inner-outer scale interaction, there is a large body of work on boundary-layer flows interacting with free stream disturbances, in which phase organization has also been observed and studied. Earlier studies investigating the boundary-layer response to sinusoidal free stream fluctuations, either stationary [14] or traveling ones [15], have found that the nonlinear effects introduced by the fluctuations were small and the statistics of the velocity field were largely unchanged even for large fluctuation amplitudes; in other words, the near-wall structure was in-phase with the unsteady freestream flow. In similar later studies [16,17], it was also observed that the boundary layer responds to sinusoidal freestream unsteadiness in a quasi-steady fashion, as the phaseaveraged flow was very similar to the steady-state flow even for relatively large frequencies; it was concluded that the turbulence production was only from the modulation of the motion. In [18] a turbulent Stokes number, $\omega D / u_{\tau}$, where $u_{\tau}$ is the skin friction velocity, was introduced for unsteady turbulent pipe flows. It was found that for low frequencies, $\omega D / u_{\tau}<1$, the turbulent structure still responds in a quasi-steady matter. For higher turbulent Stokes numbers, the boundary-layer structure will deviate from equilibrium due to an increased interaction between the structure and the imposed unsteadiness. Another parameter to study the unsteady boundarylayer response is the reduced frequency in the inner units, $\omega+=$ $\omega \nu / u_{\tau}^{2}[19]$. If $\omega+<4 \cdot 10^{-3}$, the near-wall region was found to be in phase with the unsteady freestream flow. For higher frequencies $\omega+>2 \cdot 10^{-2}$, the changes in the turbulent structure are restricted to the near-wall region of $y^{+}<20$, whereas away from the wall the structure still behaves as a quasi-steady one.

Most of the above-mentioned studies were primarily focused on the boundary-layer response to the stationary (nontraveling) unsteadiness. Only few studies, such as [15], had focused on the effect of the traveling disturbances on the boundary layer. McKeon and co-authors [20-24] have developed a resolvent analysis for turbulent flows, based on the linear amplification mechanisms associated with the linear component of Navier-Stokes equations. The idea governing the resolvent analysis is that the wall-bounded fluid flow can be represented as a summation of finite-amplitude traveling wave fluctuations over the desired range of spatial wavenumbers and frequencies about the mean velocity profile. The traveling waves are free to interact and follow rules of the welldocumented triadic scale interactions between spatial wavenumbers; 
this forcing acts as the input to the linear resolvent operator. The reader is referred to, for example, [21] for full details of the analysis. The resolvent model is built to include any number of wavenumberfrequency combinations. The ability to predict turbulent fluctuations in this manner naturally leads to the question of which wavenumberfrequency combinations are important to characterize the turbulent boundary-layer flow. Linear combinations of velocity response modes can be used to reproduce statistical and structural features of wall turbulence [22], and thus the structural phenomena become "encoded" into the flow. The analysis has highlighted the importance of the critical layer, where the local mean velocity is equal to the convection velocity of a given wave, to the localization of the response modes and therefore also the turbulent fluctuations.

With the ability to introduce different wavenumber-frequency combinations using the input forcing, it opens the possibility of including any specific internal or external forcing into the flow. With this synthetic external forcing, equations in resolvent analysis would include both the endogenous nonlinearities of the original problem as well as the exogenous applied forcing, and the added energy is manifested as a redistribution of scales consistent with triadic interactions and still extracting energy from the mean velocity profile. Based on this idea, the concept of dynamic roughness excitation of a synthetic large-scale was first introduced in Jacobi and McKeon [23] and expanded upon by Duvvuri and McKeon [25], who introduced an artificial large-scale disturbance into the flow upstream of the measurement plane to study its interaction with the small-scales in the boundary layer. The synthetic spanwise spatio-temporal mode was created using a spatially impulsive oscillating rib, which was long enough to consider the spanwise wavenumber to be zero. The rib had a height of $0.76 \mathrm{~mm}$, or about $5 \%$ of the boundary-layer thickness, with a root-mean-square (RMS) amplitude of $0.54 \mathrm{~mm}$ due to the oscillation. The frequency of oscillation was chosen to be $50 \mathrm{~Hz}$, with the intention that the combination of the amplitude and frequency would create large-scale structures with a streamwise wavelength representative VLSMs found in turbulence. The authors took hotwire data at three streamwise locations and chose to present the data at the first streamwise location, which corresponds to $x / \delta=2.7$, since the large-scale disturbance was introduced internally upstream of the testing plane and its effect decayed in the streamwise direction. The spectrogram of the premultiplied energy spectra of the velocity fluctuations was computed, and the cutoff filter was chosen so as to exclude the effect of any harmonic multiples of the forcing frequency. An analysis similar to Mathis et al. [6] was performed to split the time series into its small- and large-scale components, and the same correlations, observed in the unforced turbulent boundary layers, were also observed while studying the effect of introducing an artificial large-scale.

While studying a compressible subsonic shear layer in a wind tunnel using optical methods, Duffin [26] found optical evidence that turbulence in the high-speed boundary layer present on the upper wall of the shear-layer facility appeared as "enhanced bursts associated with the passage of coherent shear-layer structures." Because the main objective of this study was the large-scale structures in the shear layer, the details of the turbulence "bursts" in the boundary layer were left largely unexplored and motivated the work reported in this paper.

To better understand this "bursting" phenomenon, the compressible turbulent boundary layer forming on the upper wall of the shear layer wind tunnel facility was studied in a separate experimental investigation and the results are presented here. Similar to [26], the shear layer formed by the mixing of two parallel streams was mechanically forced to create deterministic large-scale vortical structures in the shear layer. The large coherent structures in the shear layer produced a concomitant unsteady pressure field, which in turn produced a variation of the effective external freestream velocity experienced by the boundary layer influencing both the large- and small-scale structures in the boundary layer. This paper examines the response of the boundary-layer structures to the external traveling shear-layer structures using hot wire anemometry. This work continues to study the relationship between the artificial large-scale disturbances created by the unsteady pressure field concomitant with the coherent shear-layer structures produced outside of the boundary layer and small-scales inside the turbulent boundary layer.

Before we continue, it is worth noticing that the present work is related, although not directly, to the effects of the large-amplitude freestream turbulence on the boundary layer, which can be treated as broad-band external traveling waves. In [27] this effect was extensively studied, and it was shown that a large, order of $10 \%$, freestream turbulence increases the velocity fluctuations throughout the boundary-layer region, and results in a larger modulating effect on the near-wall structures.

In Sec. II, the experimental setup is outlined. Although similar to the artificial disturbance in [25] created by an oscillating wall surface roughness element, the shear layer in this experiment creates a forcing external to the boundary layer instead of internal to the boundary layer. The results shown in Sec. III indicate a relationship between the artificial large-scale and the small-scales, possibly suggesting the universality of the amplitude modulation effect for different types of forcing, between forced and unforced flows, and even with varying Mach number. Conclusions are given in Sec. IV.

\section{Experimental Setup}

The compressible free shear layer facility (CSLF) located in the Hessert Laboratory for Aerospace Research at the University of Notre Dame was used to conduct the experiments. The complete description of the facility is given in [28], and so only essential details are provided here. The facility is an in-draft facility, and the air is drawn from within the room. Thus, the room total pressure and total temperature define the flow conditions at the inlet. The wind tunnel consists of two inlets, one for the high-speed flow and one for the lowspeed flow. The high-speed square inlet has a 48.5-to-1 area contraction nozzle that reduces the area from 4227 to $87.1 \mathrm{~cm}^{2}$, whereas the low-speed rectangular inlet features a slight expansion from 157.26 to $275.8 \mathrm{~cm}^{2}$. The two flows are separated by a splitter plate located $76.2 \mathrm{~mm}$ from the upper wall. The wall width is $101.6 \mathrm{~mm}$, and the test section height is linearly reduced in the streamwise direction to compensate for the total pressure losses due to the shear/boundary layers and to guarantee zero mean pressure gradient along the test section. The static pressure of the flows is matched at the splitter plate through the use of densely packed straws arranged streamwise in the expanding low-speed inlet region. The CSLF is pictured in Fig. 1a, and the schematic of experiment setup with a definition of the coordinate system is shown in Fig. 1b. Note that the vertical coordinate, $y$, will be defined as a function of the distance from the upper wall, pointing downward. All of the experiments in this paper were performed using a Mach 0.6/0.07 shear layer to ensure the reliability of hot-wire measurements for these Mach numbers.

The mixed flow goes through a throat, and the mass flow rate through the wind tunnel can be adjusted by adjusting the height of the sonic throat. The flow finally exits through a diffuser section, which is connected to one of three openings that lead to a vacuum manifold. The vacuum is produced using two Dekker Magna-Flo vacuum pumps, which can be operated individually or together. To eliminate pressure disturbances from the vacuum pumps, the airflow is choked at the throat so that the disturbances cannot propagate upstream of the throat.

The streamwise location to conduct hot-wire and unsteady pressure experiments was chosen to be the same as in the optical experiments in [26], at $x=0.43 \mathrm{~m}$. Also, similar to the approach in [26], the trailing edge voice coils at the splitter plate were mechanically actuated using a function generator, to create a deterministic synthetic large-scale disturbance in the boundary-layer flow and study its effect on the organization of small-scale turbulence. The paired vortex structures in the regularized shear layer cause unsteady pressure fluctuations, which concomitantly led to deterministic large-scale velocity fluctuations. The addition of the subharmonic guaranteed the vortex pairing mechanism that sustains the shear layer as per Oster and Wygnanski [29]. Rennie et al. [30] demonstrated the ability to robustly force this specific shear layer at high speeds using the two-frequency approach, and more details 


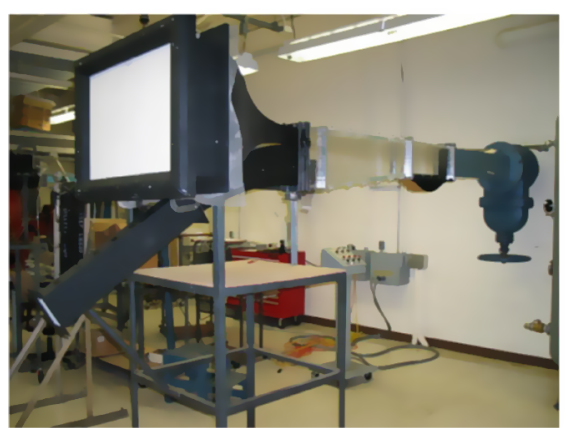

a)

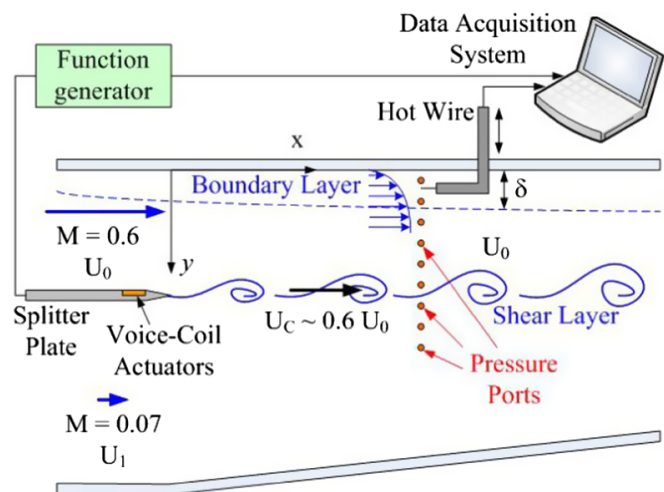

b)

Fig. 1 a) Compressible shear layer facility, photograph. b) Schematic of the experimental setup, including the system of coordinates and flow parameters.

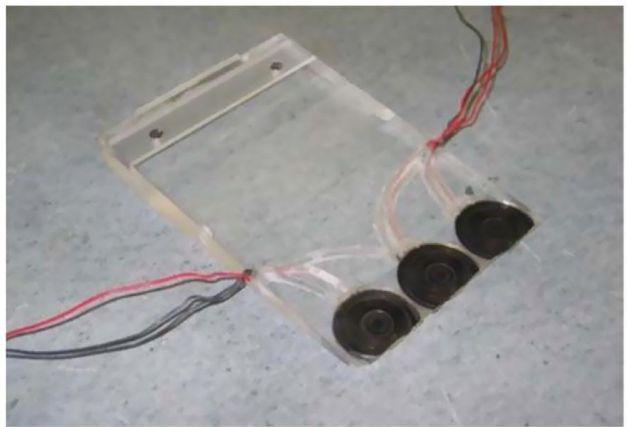

a) b)

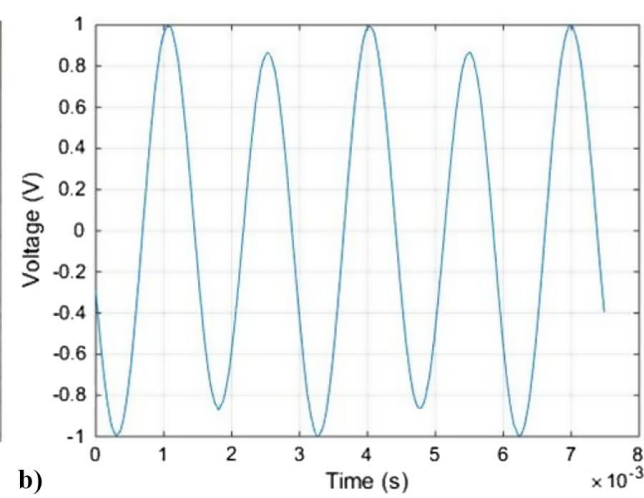

Fig. 2 a) Photograph of voice coil actuators on the splitter plate, and b) sample of forcing signal. about the forcing can be found in their paper. For the presented experiment, the shear layer was forced using voice-coil actuators on the splitter plate at a frequency of $f_{\text {force }}=675 \mathrm{~Hz}$. A subharmonic at half of the forcing frequency and one-tenth amplitude was added to the forcing signal to control the vortex pairing mechanism, which serves to regularize the shear layer. The voice coil actuators and the forcing signal are shown in Fig. 2. Main shear layer parameters are provided in Table 1 .

Because the focus of this work was to study the turbulent boundary layer, the velocity results are presented either in the outer units, using the high-speed free-stream velocity, $U_{0}$, and the boundary-layer thickness, $\delta$, or in the inner units, using the skin friction velocity, $u_{\tau}$. These quantities, as well as other boundary-layer parameters, are presented in Table 2 .

A Dantec single-wire miniature boundary-layer hot-wire probe (type 55P15) was used to acquire time series of velocity throughout the boundary layer at the chosen streamwise location. The sensor had a wire of $5 \mu \mathrm{m}$ diameter, $1.25 \mathrm{~mm}$ length $\left(l^{+}=416\right)$, and an approximate resistance of $3.5 \mathrm{ohms}$, and was made out of platinumcoated tungsten. With a large $l^{+}$value there is a likelihood of significant spatial attenuation of the smallest scales [31], but because it was difficult to obtain measurements very close to the wall due to the relatively thin turbulent boundary layer, all of the results represent points in the outer boundary layer and higher where attenuation due to the wire length is likely to be minimized; however, the smaller scales will continue to be attenuated to some degree. The wire was operated by an AA Systems anemometer using an overheat ratio of 1.7. Because of the compressible nature of air in the high-speed region, a modified King's Law approach, $E^{2}=A+B(\rho U)^{m}$, that accounted for flow densities was used to acquire a time series of velocities from a time series of voltages. During calibration, the hot wire was placed in the freestream, the freestream Mach number was varied between 0 and 0.6 , and the resulting voltages were recorded. The Mach numbers were converted to the corresponding mass flux, $\rho U$, using the isentropic assumption, and the calibration constants $A$, $B$, and $m$ were calculated. As the total temperature is approximately constant in the freestream and inside the boundary layer, this approach will introduce only small errors in Mach/velocity measurements in this Mach number range [32]. A postcalibration was performed to account for drift, and a linear interpolation between the pre- and postcalibration was used to process the data.

During the measurements, time series of voltages were first converted to a time series of mass flux, from which the instantaneous Mach number and streamwise velocity were calculated using the

Table 1 Shear layer parameters

\begin{tabular}{lcccc}
\hline \hline $\begin{array}{l}\text { High-speed/low-speed free-stream } \\
\text { Mach numbers, } M_{0} / M_{1}\end{array}$ & $\begin{array}{c}\text { Convective Mach number of the shear layer, } M_{c} \\
\text { (see text for definition) }\end{array}$ & Static pressure, $P_{s}$ & Total temperature, $T_{0}$ & $\begin{array}{c}\text { Shear layer forcing } \\
\text { frequency, } f_{\text {force }}\end{array}$ \\
\hline $0.6 / 0.07$ & 0.27 & $78 \mathrm{kPa}$ & $293 \mathrm{~K}$ & $675 \mathrm{~Hz}$ \\
\hline \hline
\end{tabular}

Table 2 BL parameters

\begin{tabular}{lccccccc}
\hline \hline $\begin{array}{l}\text { BL thickness, } \delta \text { (based on } \\
99 \% \text { of } U_{0} \text { ) }\end{array}$ & $\begin{array}{c}\text { Displacement } \\
\text { thickness, } \delta^{*}\end{array}$ & $\begin{array}{c}\text { Momentum } \\
\text { thickness, } \theta\end{array}$ & $\begin{array}{c}\text { Free-stream } \\
\text { velocity, } U_{0}\end{array}$ & $\operatorname{Re}_{x}$ & $\operatorname{Re}_{\delta}$ & $\begin{array}{c}\boldsymbol{R e}_{\theta} \\
u_{\tau} \text { (estimated, } \\
\text { see text) }\end{array}$ & $\begin{array}{c}\operatorname{Re}_{\tau} \text { (estimated, } \\
\text { see text) }\end{array}$ \\
\hline $19.7 \mathrm{~mm}$ & $2.9 \mathrm{~mm}$ & $2.7 \mathrm{~mm}$ & $209 \mathrm{~m} / \mathrm{s}$ & $4.95 \times 10^{6}$ & $2.26 \times 10^{5}$ & 31,600 & $\sim 6.7 \mathrm{~m} / \mathrm{s}$ \\
\hline \hline
\end{tabular}


isentropic relation. Because the region of interest was the boundary layer on the upper wall (in the high-speed region), a 61-point wallnormal traverse of hotwire data was taken. For the experimental hotwire data, the sampling time was $10 \mathrm{~s}$, or about 120,000 eddy turn over times (estimated as $\delta / U_{0}$ ), and a sampling frequency of $f_{s}=$ $80 \mathrm{kHz}$ was chosen. This sampling frequency corresponds to the nondimensional sample interval of $\Delta t^{+}=\left(1 / f_{s}\right) u_{\tau}^{2} / \nu=34$. For this relatively large value, high frequencies in the near-wall will not be properly resolved and will potentially be aliased. However, as mentioned before, all the measurements were performed in the outer region, where the frequencies should be lower in general, and so this high-frequency misrepresentation is not expected to affect the results.

An array of 22 XTL/XTEL-140 Series Kulite pressure transducers was placed on the side wall of the wind tunnel at the same streamwise position, as the hot wire measurements (see Fig. 1b). The transducers were spaced $0.35 \delta$ apart in the wall-normal direction, with the first one being $0.2 \delta$ away the wall. The transducers were screwed into place and secured with pressure-fit O-rings such that the sensor face was flush with the inside of the wind tunnel. A sampling time of $15 \mathrm{~s}$ at a lower sampling frequency of $20 \mathrm{kHz}$ was used to acquire time series of fluctuating static pressures. Additional static taps were installed along the tunnel side wall in the streamwise direction and monitored during the experiments to ensure the lack of a streamwise pressure gradient.

The forcing signal and the voltages from both the hot-wire anemometry and the unsteady pressure sensors were synchronized and collected simultaneously in order to apply the phase-locked analysis.

\section{Results}

The mean velocity profile of the flow as a function of position from the wind tunnel upper wall is plotted in Fig. 3a. The plot shows a typical shear layer velocity profile between $y \overline{/ \delta}=2$ and 7 with a uniform region of high-speed flow on one side of the shear layer, between $y / \delta=1$ and 2 . The turbulent boundary layer is also clearly present on the wall. The turbulent boundary layer in Fig. 3a is flatter than a canonical boundary layer, indicating that the boundary layer observed in the experiment is not a canonical turbulent boundary layer. The displacement thickness and the momentum thicknesses of the boundary layer were calculated using the velocity profiles and are given in Table 2 . The shape factor, $H=\delta^{*} / \theta$, was determined to be 1.05. For comparison, a typical value of the shape factor for an incompressible, zero-pressure gradient turbulent boundary layer for similar Reynolds numbers is about 1.3 [33]. The deviation from the canonical boundary-layer velocity profile is not surprising, because the boundary layer is constantly experiencing local shear-layerrelated adverse and favorable pressure gradients. The flattening of the mean velocity profile due to alternating pressure gradients was shown in the experiments in [34], which used suction and blowing through an upstream slot to study its effect on the turbulent boundary layer. A similar reduction of $\mathrm{H}$-factor was also observed in boundary layers, subject to high levels of freestream turbulence [27], and was attributed to an increased mixing in the boundary layers.

Friction velocity, $u_{\tau}$, was estimated using either $\delta^{*}$ or $\theta$ via ColesFernholz approximations [35] and were found to be similar, 6.8 and $6.65 \mathrm{~m} / \mathrm{s}$, respectively. Using the average value between these two quantities, $u_{\tau}=6.73 \mathrm{~m} / \mathrm{s}, R e_{\tau}$ was estimated to be $\sim 7600$. The mean velocity profile inside the boundary layer in the inner units is presented in Fig. 3b. The theoretical law-of-the-wall velocity profile $U^{+}=1 / \kappa^{*} \ln \left(y^{+}\right)+C$ is also plotted in Fig. $3 \mathrm{~b}$ for $C=-1.5$. Because the studied boundary layer is not a canonical one, the $C$-constant is expected to differ from the typical value of 4.2 [36]. It is interesting to note that the boundary layer, despite being a noncanonical one, appears to exhibit a $\log$ region below $y^{+}<1000$ or $y / \delta<0.13$, and the upper bound of the log region agrees with the one for the canonical boundary layer, which is $y^{+}=0.15 R e_{\tau} \sim 1100$ [6]. Of course, having only three measurement points inside the log region, the statement about the log region should be treated with some degree of caution.

The $y$-location of the splitter plate is marked as a solid line at $y / \delta=3.86$ away from the wall in Fig. 3a, and approximately corresponds to the center line of the shear layer. The convective speed, $U_{C}$, of the large-scale structures in the shear layer can be estimated using isentropic assumption [37] as,

$$
U_{c}=\frac{a_{1} U_{0}+a_{0} U_{1}}{a_{0}+a_{1}}
$$

where $U_{0}$ and $U_{1}$ represent the high and low speeds, respectively, and $a_{0}$ and $a_{1}$ are the speeds of sound for the high and low speed flows, respectively. This equation provides a good approximation (within few percent) of the convective speed, if the convective Mach number, $M_{c}=\left(U_{0}-U_{1}\right) /\left(a_{0}+a_{1}\right)$ is less than 0.4 [38]; $M_{c}$ is 0.27 for this study, indicating that the turbulence can be considered incompressible [39]. The convective speed for the Mach 0.6/0.07 shear layer is about $115 \mathrm{~m} / \mathrm{s}$ or $0.56 U_{0}$. This speed is close to the value of the local speed of $125 \mathrm{~m} / \mathrm{s}$, or $0.6 U_{0}$, at the location of the splitter plate. Also, the velocity ratio of the shear layer, defined as, Ratio $=\left(U_{0}-U_{1}\right) /\left(U_{0}+U_{1}\right)$, is approximately 0.77 . Finally, knowing the convective speed and the forcing frequency, the spatial separation between the consecutive shear layer structures was computed to be $0.17 \mathrm{~m}$ or $8.6 \delta$.

RMS of the mean-removed pressure fluctuations at each wallnormal location in the wind tunnel was calculated and is shown in Fig. 4a. As expected, the largest RMS of pressure occurs in the area of
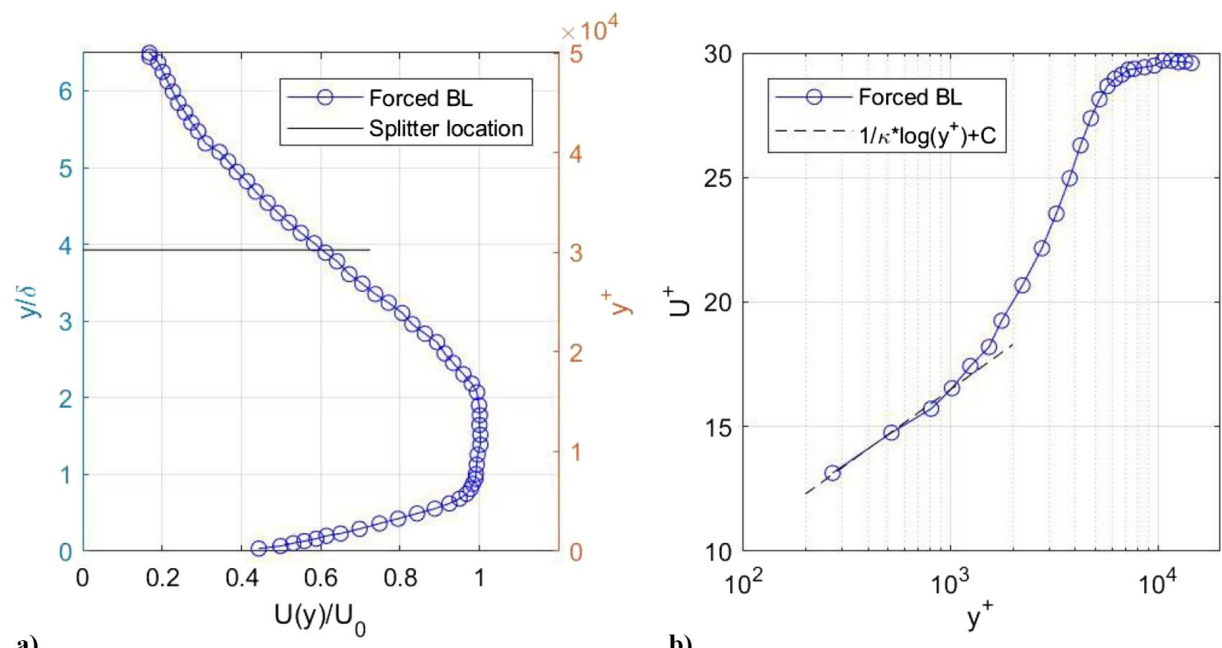

Fig. 3 a) Mean velocity profiles, normalized by the free-stream velocity in both outer $(y / \delta)$ and inner $\left(y^{+}\right)$units. The location of the splitter plate is marked as a solid line. b) Mean velocity profile inside the boundary layer in inner units, along with the theoretical velocity profile in the log region, $U^{+}=1 / \kappa * \ln \left(y^{+}\right)+B, \kappa=0.384, C=-1.5$. 


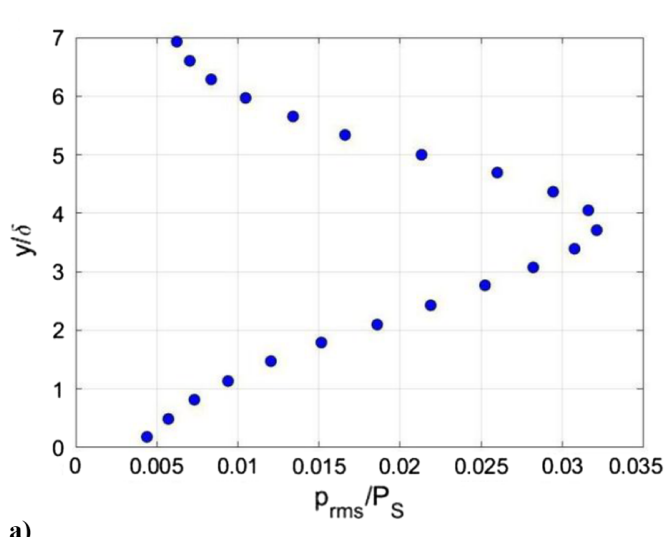

a)

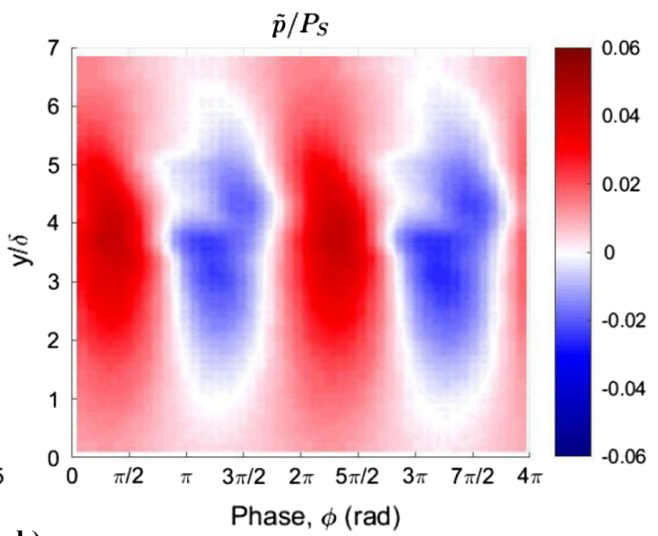

b)

Fig. 4 a) RMS of mean-removed pressure, $p_{\text {rms }}$, normalized by static pressure $\boldsymbol{P}_{S}$. b) Phase-averaged modal pressure field, $\tilde{p}(y, \varphi)$, normalized by $\boldsymbol{P}_{S}$.

the shear layer, near $y / \delta=3.8$, as the presence of coherent structures causes large fluctuations in instantaneous static pressure. The pressure fluctuations in the boundary layer are small, $p_{\text {rms }} / P_{S}<0.01$.

The mean-removed pressure fluctuations at each wall-normal location in the wind tunnel were calculated from the Kulite measurements. The time series of fluctuating static pressures at each measurement location was phase-locked with respect to the input forcing signal, $t=n / f_{\text {force }}+\phi /\left(2 \pi f_{\text {force }}\right)$ where $n$ is integer number and $\phi=[0, \ldots, 4 \pi]$ is the phase, and averaged over a twocycle forcing signal with the phase range of 0 to $4 \pi$ divided into $N=50$ equally sized phase bins to obtain the triple decomposition, similar to [40],

$$
p(y, t)=\bar{p}(y)+\tilde{p}(y, \varphi)+p^{\prime}(y, \varphi ; n)
$$

The term with a bar represents a conventional time averaging; the term with tilde represents the phase-averaged, large-scale component (later it will be called a modal component) of the pressure directly arising from the pressure decreases or pressure "wells." The term $p^{\prime}$ represents the residual fluctuations at each phase. It is easy to demonstrate that the relation between mean-removed $P_{\text {rms }}$, shown in Fig. 4a, and the triple decomposition components is $p_{\text {rms }}^{2}(y)=$ $\left\langle\tilde{p}^{2}(\overline{y, \varphi})\right\rangle_{\phi}+\left\langle\left[p^{\prime}(y, \varphi ; n)\right]^{2}\right\rangle_{\phi, n}$, where angular brackets indicate averaging over $\phi$ and $n$.

The phase-locking procedure was performed for $n=10,125$ events, coincident with forcing periods. A surface plot, $\tilde{p}(y, \varphi) / P_{S}$, showing the ensemble-averaged magnitude of modal pressure field at measurement locations on the side wall is shown in Fig. 4b. Two pressure wells at $\phi=5 \pi / 4$ and $13 \pi / 4$, associated with the passing of the forced shear layer coherent structures, separated by two regions of pressure increase at $\phi=\pi / 4$ and $9 \pi / 4$, can be clearly observed. The convecting shear layer coherent structures create a concomitant unsteady pressure field in the flow; this is experienced in the boundary layer as a variation of the local freestream velocity. The pressure field inside the boundary layer is in phase with the pressure field inside the shear layer structures; thus the modal pressure inside the shear layer will be used as a reference pressure signal later in this paper.

The time series of velocities were also postprocessed using the same phase-locking algorithm as the pressures, which resulted in the triple decomposition,

$$
u(y, t)=\bar{U}(y)+\tilde{u}(y, \varphi)+u^{\prime}(y, \varphi ; n)
$$

The term $\tilde{u}(y, \phi)$ is related to the slow-varying deviation of the phase-locked averaged velocity from the time-averaged velocity; in this paper it will be referred as modal velocity, because it is due to the periodic (modal) external changes in velocity and pressure. The term $u^{\prime}(y, \phi ; n)$ will be called residual phase-locked turbulence. Analyzing the turbulence in this manner decomposes the time series similarly to [25], where the signal was phase-locked over one cycle to their single-frequency, purely sinusoidal input forcing.

The overall turbulence intensity, $u_{\mathrm{rms}}$, was calculated at each point in the boundary layer and, after normalization by the freestream velocity, is presented in Fig. 5a as a line with circles. Because the flow was forced with a sinusoidal signal, the "turbulence intensity" of the full velocity signal is misleading, as it includes the contribution from both the modal (deterministic) velocity and the truly turbulent component. Using Eq. (2) it is straightforward to show that $u_{\mathrm{rms}}^{2}(y)=\left\langle\tilde{u}^{2}(y, \varphi)\right\rangle_{\phi}+\left\langle\left[u^{\prime}(y, \varphi ; n)\right]^{2}\right\rangle_{\phi, n}$, where brackets indicate averaging over $\phi$ and $n$. A proper way to calculate turbulence levels is to exclude the modal velocity component and compute the residual turbulent intensity,

$$
u_{\mathrm{rms}}^{\prime}(y)=\left(\left\langle\left[u^{\prime}(y, \phi ; n)^{2}\right]\right\rangle_{\phi, n}\right)^{1 / 2}
$$

The residual turbulent intensity profile, normalized by $U_{0}$, is shown in Fig. $5 \mathrm{a}$ as a solid line. The increase of the residual turbulence intensity near the wall through the work of Reynolds stresses against a mean velocity gradient is evident in the plot. Maximum residual turbulent intensity reaches about $7 \%$ of the freestream speed, similar to turbulent levels in canonical boundary layers. It can also be seen that the CSLF is a relatively quiet wind tunnel, with the freestream turbulence of approximately $1 \%$ of the tunnel speed.

For the canonical BL, the near-wall peak in turbulence is near $y^{+} \sim 15$ [41], and this location is largely independent of $R e_{\tau}[\underline{6}, \underline{26}]$. As mentioned in the Introduction, at high $\mathrm{Re}_{\tau}$, an additional outer peak appears in the turbulent profile [6], located in the middle of the $\log$ region at $y_{\mathrm{OL}}^{+} \sim 3.9 R e_{\tau}^{1 / 2}[\underline{6}, \underline{42}]$. To see how the studied boundary layer compares to the canonical one, the residual turbulence intensity in the inner units is plotted in Fig. 5b. There is no data below $y^{+}<200$, but the near-wall peak in the turbulence intensity is expected to be present [27]. The location of the maximum turbulent intensity is at $y / \delta=0.25$ or $y^{+} \sim 2000$ (see Fig. 5b), indicating that the peak is outside of the log region and in the wake region. This is in contrast with the canonical boundary layer at the same $R e_{\tau}$, where the outer peak should be in the log region, $y_{\mathrm{OL}}^{+} \sim 340$. The shift in the location of the turbulence peak away from the wall is a result of the interaction of the boundary layer with the external traveling variation of the freestream speed. Note that similar observations were made in [27], where in the case of high levels of the freestream turbulence, the outer turbulent peak was also found farther away from the wall, at $y / \delta \sim 0.31$.

An example of a modal velocity and residual turbulence component is shown in Fig. 6 a for one $y$ location, $y / \delta=0.25$, where the overall turbulence fluctuations are the largest (see Fig. $\underline{5 a}$ ). In Fig. $6 \mathrm{a}$, the solid line represents the modal velocity component, $\tilde{u}$ normalized by $U_{0}$. The phase-locked modal velocity is sinusoidal due to the voice-coil actuation. Also, it has a zero-mean across all phases, since the time-averaged value, $\bar{u}$, is removed. Note that the presence 


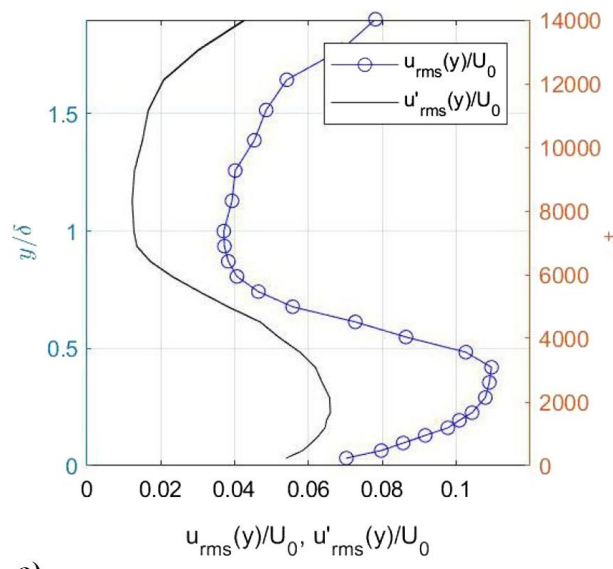

a)

Fig. 5 a) Normalized overall turbulence intensity, $u_{\mathrm{rms}} / U_{0}$, and residual turbulent intensity, $u_{\mathrm{rms}}^{\prime} / U_{0}$, for the forced boundary layer as a function of $y / \delta$ and $y^{+}$. b) Residual turbulent intensity in inner units, $u_{\mathrm{rms}}^{\prime} / u_{\tau}$ vs $y^{+}$.

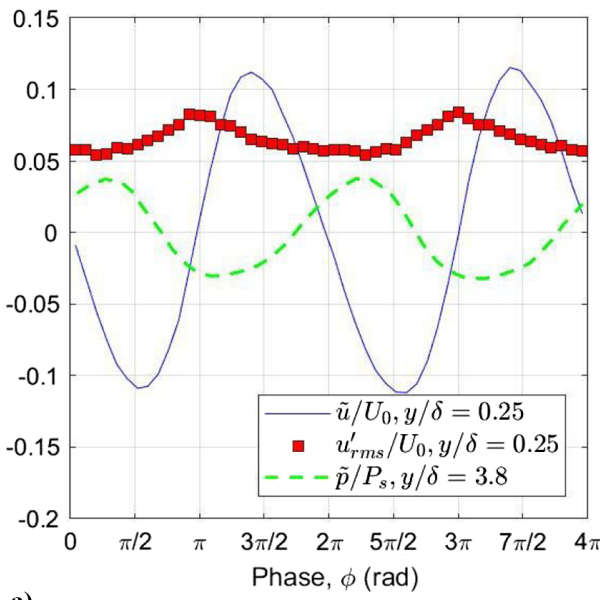

a)

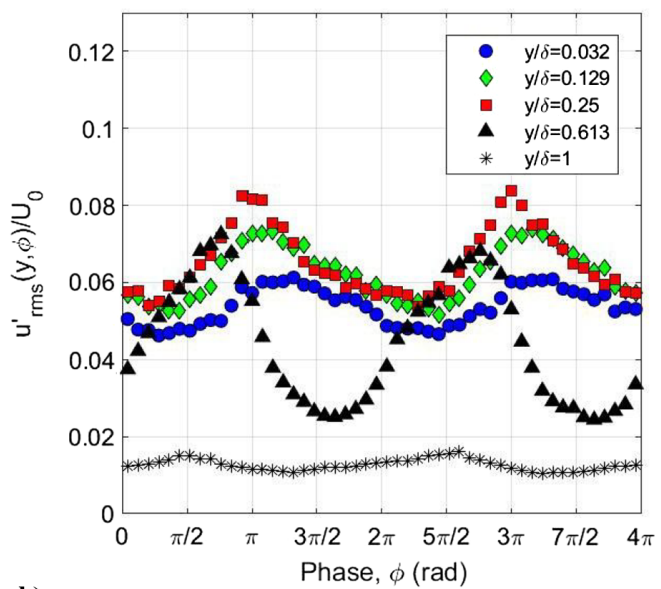

b)

b)

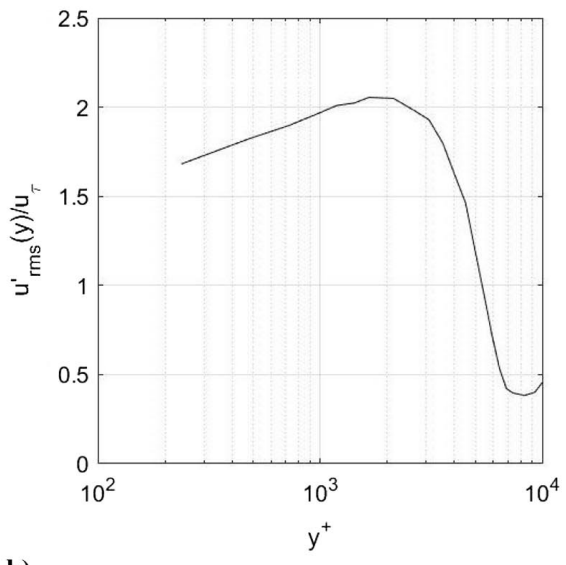

Phase, $\phi(\mathrm{rad})$

Fig. 6 a) Phase-locked average (modal) velocity and residual turbulence intensity, defined in Eq. $(\underline{3})$, at $y / \delta=0.25$, and the modal pressure, $\tilde{p}(y, \varphi) / P_{s}$, at $y / \delta=3.8$. b) The residual turbulence intensities at various wall-normal locations.

of the subharmonic in the forcing signal does not have any significant effect on the phase-averaged velocity field, as this subharmonic simply stabilizes the pairing location in the shear layer [30]. For reference, the modal pressure inside the shear layer at $y / \bar{\delta}=3.8$, normalized by the static pressure, is also presented in Fig. 6a. There is a clear relationship between the modal velocity and the pressure, with regions of positive pressure roughly corresponding to a negative velocity fluctuation and vice versa.

The red squares in Fig. 6a represent the RMS of the phase-locked residual turbulence intensity in each phase and normalized by the freestream speed, $u_{\mathrm{rms}}^{\prime}(y, \varphi) / U_{0}$, at the same $y / \delta=0.25$ location. There is a marked increase (from about $5 \%$ to close to $8 \%$ ) in the phase-locked turbulence intensity at certain phase angles (namely, $\pi$ and $3 \pi$ ) and indicates a certain (phase) arrangement in the smallscales, which we will define as turbulence amplification. This amplification is phase-locked with the modal velocity, which is in turn related to the passing of coherent structures outside of the boundary layer.

The residual turbulence intensity, normalized by the free-stream speed, is plotted for four different wall-normal locations in Fig. 6b. It can clearly be seen from Fig. $6 \mathrm{~b}$ that as the wall-normal location increases, the maximum values of the phase-locked turbulence intensity, as well as the difference between the maximum and the minimum values of the phase-locked turbulence increase for $y / \delta<0.62$. Farther away from the wall, they start decreasing. Near the wall at $y / \delta=0.032$, the turbulence intensity goes as low as 0.05 and reaches the maximum value of 0.06 , whereas at $y / \delta=0.613$ the turbulence intensity values vary between 0.025 and 0.075 . As the wall-normal location increases to a value of $y / \delta=1$, the magnitude of the turbulence intensity decreases significantly; nevertheless, it is noteworthy that the peak in amplitude is now anticorrelated in phase with the first two locations shown. The peak in the turbulent activity occurs at a different phase-angle with increasing wall-normal distance. At the edge of the boundary layer, $y / \delta=1$, for example, the slight amplitude increase occurs closer to $\pi / 2$; however, closer to the wall at $y / \delta=0.032$, the amplitude increase occurs at $3 \pi / 2$, representing a phase change of nearly $\pi$ radians, relative to the edge region. This variation of phase with the peak in turbulence increase is a reflection of the modulation effect and can be represented through the use of a correlation coefficient, as will be shown later in this paper.

To better visualize the effect of the external forcing on the boundary layer, the phase-locked modal velocities, $\tilde{u}(y, \phi)$, were calculated for the entire set of measurements in the boundary layer and the resulting contour plot is shown in Fig. 7a. The reference phase-locked modal pressure, $\tilde{p}(\varphi) / P_{s}$, inside the shear layer is also plotted for comparison in Fig. 7a to indicate the locations of the shear layer structures (with low-pressure regions). The magnitude of this sinusoidal velocity component inside the boundary layer is worth noting. As the wall-normal location increases, the magnitude of the sinusoidal modal velocity component increases until a point and then decreases toward the outer edge of the boundary layer. Most of the variation within the modal component is concentrated in a region between $y / \delta \approx 0.2-0.6$, with values close to $10 \%$ of the free-stream velocity. As it was already observed in Fig. 6 a, the regions of lower pressure correspond to higher modal velocities and vice versa when phase-locked to the forcing signal. The phase-locked plot shows that there is a small shift in the phase of the modal velocity between $y / \delta=1$ and $y / \delta=0.5$. Thus, the modal velocity inside the 


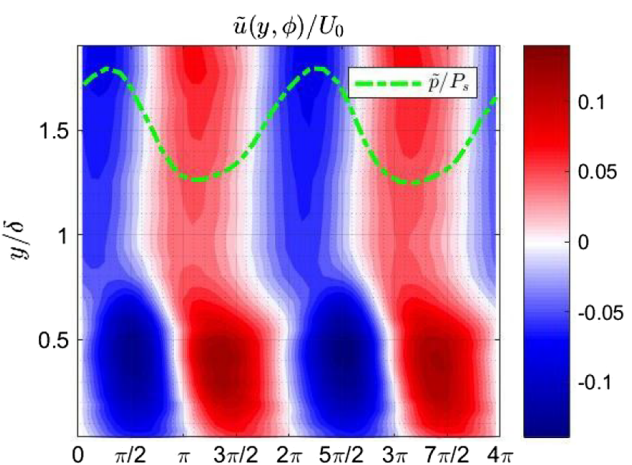

a)

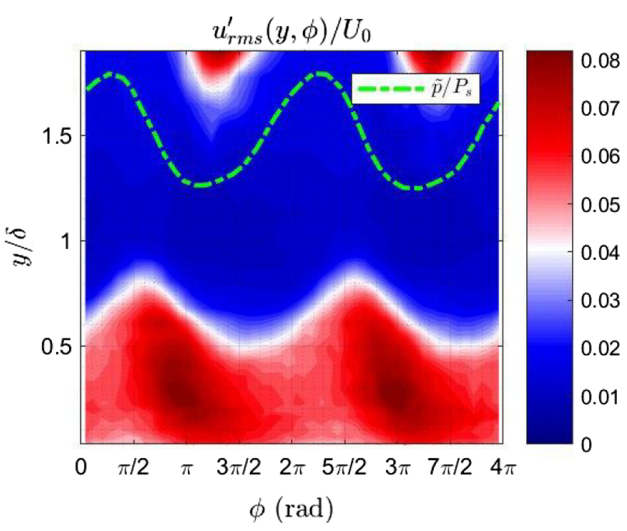

c)
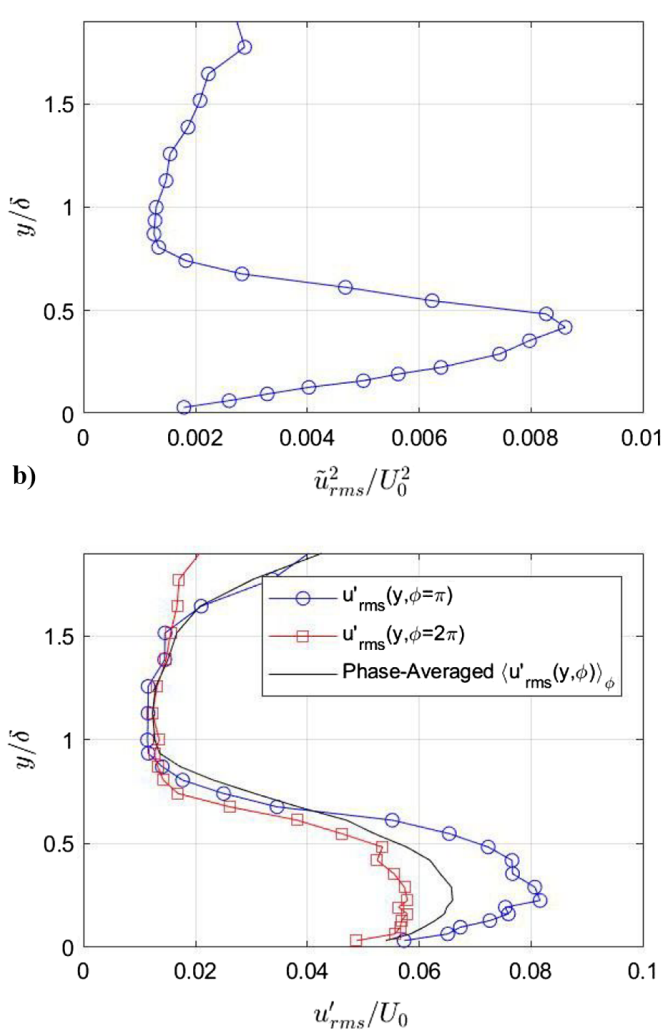

d)

Fig. 7 a) Phase-locked modal velocity as a function of the wall-normal distance, $\tilde{u}(y, \varphi) / U_{0}$. The modal pressure inside the shear layer, $\tilde{p}(\varphi) / P_{S}$, is also presented as a dashed line to indicate the locations of the vortical structures (low values of pressure). $b$ ) The wall-normal distribution of energy of the modal velocity component. c) Contour plot of normalized residual phase-locked turbulence intensity, $u_{\mathrm{rms}}^{\prime}(y, \varphi) / U_{0}$.d) $u_{\mathrm{rms}}^{\prime}(y, \varphi) / U_{0}$-profiles for $\varphi=\pi$ and $2 \pi$.

boundary layer has a small phase lag relative to the modal velocity outside of the boundary layer, most probably due to the intermittent region. Figure $\underline{7 a}$ also highlights the deterministic quality of the regularized shear layer, or $y / \delta>1.5$.

The energy contained within the externally imposed large-scale modal velocity component was calculated as $\tilde{u}_{\text {rms }}^{2} / U_{0}^{2}$, where $\tilde{u}_{\text {rms }}$ is the phase-averaged RMS of the modal velocity component, and is plotted as a function of the wall-normal position in Fig. 7b. From Fig. $7 \mathrm{~b}$, the peak in modal energy occurs at a wall-normal location of approximately $y \sim 0.4 \delta$.

In a similar matter, the normalized levels of the residual turbulence intensity, $u_{\mathrm{rms}}^{\prime}(y, \varphi) / U_{0}$, are plotted for the entire measurement region and presented in Fig. 7c, along with the reference modal pressure. Localized inclined regions of the amplified turbulence intensity can be seen near phase values of $\pi$ and $3 \pi$. The large-scale, shear-layer-related velocity fluctuations can also be seen for $y$-values larger than $1.6 \delta$, coinciding with the lower-pressure regions. There is a clear phase variation of the residual turbulence with increasing wallnormal distance. Near the wall, the turbulence intensity is almost directly in phase with the large-scale external disturbance. Closer to the boundary-layer edge, the turbulence tends to be slightly ahead of the large-scale disturbance.

Figure $7 \mathrm{~d}$ shows the profiles of $u_{\mathrm{rms}}^{\prime}(y, \varphi) / U_{0}$ for two selected phases, $\phi \equiv \pi$, where the residual turbulence intensity reaches the maximum value, and $\phi=2 \pi$, where the residual turbulence intensity is at minimum inside the boundary layer. The largest phase-locked values of turbulent intensity occur for $\phi=\pi$ in a region of $y / \delta$ between 0.2 and 0.4 . For canonical boundary layers, the maximum turbulence intensity is very near the wall; however, for this externally forced boundary layer it is shifted away from the wall. Note from Fig. $3 \mathrm{~b}$ that the mean velocity at this wall-normal region is between $0.6 U_{0}$ and $0.75 U_{0}$, which is close to the convective speed of the external disturbances by the shear layer, $U_{C} \sim 0.6 U_{0}$. The matched speeds indicate the existence of a critical layer at this region; as mentioned in the Introduction, these critical layers were shown to provide the main amplification mechanism in turbulent flows and are an important feature in resolvent analysis $[21,24]$.

In [43] the energy transfer between the mean velocity, $\bar{u}$ large-scale periodic (organized) velocity component, $\tilde{u}$, and the small-scale turbulence, $u^{\prime}$, was derived. It was demonstrated that, for spatially homogeneous flow $(\partial U / \partial x=0)$, the turbulence production is proportional to $\overline{-\left\langle u^{\prime} u^{\prime}\right\rangle \partial \tilde{u} / \partial x}$, where the overbar denotes the time averaging and the angular brackets denote phase averaging. From here it follows that the production of small-scale (residual) turbulence should increase in the regions with a negative spatial gradient of the modal velocity, $\partial u_{\%} / \partial x<0$. Recalling that from Taylor's "frozen" turbulence hypothesis, $\partial / \partial x=-(1 / \bar{U}) \partial / \partial t$, and so the turbulence should be the largest where the temporal (phase) derivative of the local modal velocity is positive and the smallest where the phase derivative of the modal velocity is negative. Indeed, $u_{\mathrm{rms}}^{\prime}$ reaches the maximum at phase values of $\pi$ and $3 \pi$ (see Figs. 7c and 7d), where the modal velocity has a large positive phase gradient (see Fig. 7a). Likewise, at the phase of $2 \pi$, the modal velocity has the largest negative phase gradient and the turbulence reaches the smallest values at this phase (see Figs. 7c and 7d).

The boundary layer has elevated levels of turbulence, whereas the freestream has lower turbulence fluctuations. So, the boundary between the "low" and "high" turbulence can be interpreted as a "edge" of the boundary layer. From Fig. 7c, it can be observed that the boundary layer is the thickest near phases of $\pi / 2$ and $5 \pi / 2$, whereas it is the thinnest at $3 \pi / 2$ and $7 \pi / 2$. Interestingly, these locations do not correspond to either maxima or minima of the freestream external velocity at $\pi$ and $3 \pi$ (see Fig. 7a). Rather, these locations of the thickest and the thinnest boundary layer line up fairly well with the local minima and maxima of the phase-locked modal velocity, $\tilde{u}$, within the turbulent boundary layer. They also correlate with the regions of the external flow accelerations and decelerations, which are linked to the freestream pressure gradients. This implies that the boundary thickness depends on the pressure gradient variation. 


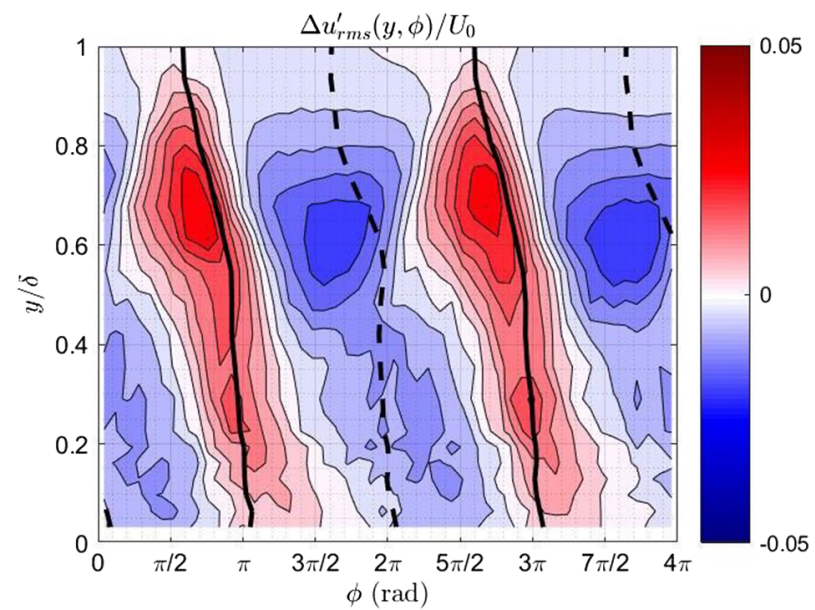

Fig. 8 Mean-removed residual turbulence intensity, $\Delta u_{\mathrm{rms}}^{\prime}(y, \phi) / U_{0}$. Superposed are lines of the positive (thick solid lines) and negative (thick dashed lines) zero crossings of the modal component, $\tilde{u}$.

A quasi-linear approximation, proposed in $[10,11]$, attempts to explain the modulation of the near-wall structure by the large-scale structures by assuming that the near-wall region behavior depends on the large-scale varying velocity outside of the region. Consequently, mean-removed and properly filtered variation in the near-wall turbulence intensity should be proportional to, or completely in phase with, the slowly varying large-scale velocity [44]. For the presented work, it would imply that $\Delta u_{\mathrm{rms}}^{\prime}(y, \phi) /\left\langle u_{\mathrm{rms}}^{\prime}(y, \phi)\right\rangle_{\phi} \tilde{u}(y, \phi) / \bar{U}(y)$ in the near-wall region, where

$$
\Delta u_{\mathrm{rms}}^{\prime}(y, \phi)=u_{\mathrm{rms}}^{\prime}(y, \phi)-\left\langle u_{\mathrm{rms}}^{\prime}(y, \phi)\right\rangle_{\phi}
$$

is the mean-removed residual turbulence intensity, shown in Fig. $\underline{8}$. Unfortunately, the presented experiments did not include the nearwall region, below $y^{+}<200$, and the validity of the quasi-steady relation for the externally forced boundary layer cannot be investigated directly.

In $[44,45]$ a criterion of a temporal positive zero crossing of the large velocity fluctuations was used to extract conditionally averaged zero-mean fluctuation levels of the small-scale structures; zero crossing corresponds to the internal shear layer between high-speed and low-speed zones. It was concluded that in the near-wall region, the results generally support the quasi-steady model. Away from the wall, additional increased turbulence level was found to be in the center of the log region; the increase also coincides with the positive zero crossing. It was argued that in this region the turbulence increase is due to a scale rearrangement, rather than a modulation. Finally, in the intermittent region, a negative correlation between the large and the small structures was observed.

In the present study, $\Delta u_{\mathrm{rms}}^{\prime}(y, \phi) / U_{0}$ in Fig. $\underline{8}$ represents the zeromean residual turbulence intensity. The zero crossings for modal velocity, located near $\phi=\pi, 3 \pi$ (positive crossing) and near $2 \pi, 4 \pi$ (negative crossing), are also plotted in Fig. 8 as thick solid and dashed lines, respectively. The regions of increased turbulence near $y / \delta=$ 0.3 at the phase values of $\pi$ and $3 \pi$ are clearly correlated with the positive zero crossings of the modal component, consistent with the observations in $[44,45]$ and the turbulence production mechanism, discussed earlier. An additional large increase in $\Delta u_{\mathrm{rms}}^{\prime}(y, \phi)$ at $y / \delta=0.7$ is related to the intermittency region; these increases lead in time the positive zero crossings, also in agreement with findings in $[44,45]$. Thus, the conceptual arrangement between the large-and the small-scale structures in the canonical boundary layer, proposed in [44], is believed to be also valid for the externally forced boundary layer.

It is interesting to relate the presented results with the boundary-layer response to unsteady but stationary freestream distortions, imposed on the mean flow. One of the important parameters used to study this response is the reduced frequency in inner units, $\omega^{+}=\omega V / u_{\tau}^{2}$ [19]. Using the value of forcing frequency, $\omega^{+} \sim 1.7 \times 10^{-3}$ for this study; for this small value, the near-wall region should be in phase with the unsteady freestream flow [19]. Figure 8 shows that the increase in turbulence levels near the wall is between the phase values of $\pi$ and $3 / 2 \pi$, which indeed is in phase with the increase in the freestream speed (see Fig. 7a). Also, the Strouhal number for this study is low, $\omega(2 \delta) / U_{0} \sim 0.8$, implying that a quasi-steady turbulence model can be used to predict the flow response [18]. The turbulence production mechanism, presented earlier, is in fact based on the quasi-steady model. The agreement between the predicted and the observed locations in the increases in turbulence does indicate that the quasi-steady turbulence model can be used at this low forcing frequency.

In the original work by Duffin [26], where the "bursts" related to higher levels of turbulence were observed optically, the optical interrogation region included both the boundary layer and the shear layer, which provided the external forcing of the boundary layer. Therefore, it was only a conjecture made by Duffin that these higher levels of turbulence-related optical distortions resided in the boundary layer. But, if the velocity statistics inside the boundary layer are known, optical distortions due to the boundary layer can be calculated using a "linking" equation [46], combined with strong Reynolds analogy (SRA); this approach was found to provide correct levels of optical distortions for canonical $[47,48]$ and nonadiabatic wall [49] boundary layers. This approach was used to predict optical distortions due to the externally forced boundary layer in [50], and it was demonstrated that if the interaction between the pressure and the velocity fields is properly accounted for, the predicted optical distortions match the experimentally observed ones. Thus, there is sufficient reason to believe that the boundary-layer "bursts" observed optically by Duffin [26] are indeed manifested as regions of amplified turbulence and are being captured in this work using hotwire anemometry.

It is obvious that the work of Duvvuri and McKeon [25] most closely resembles the current work, as in both cases the turbulent boundary layer is excited at a specific forcing frequency and a synthetic large-scale is created. Following [25], a spectrogram of the premultiplied energy spectra was computed and is shown in Fig. 9a. The spectrogram shows a clear spike at the forcing frequency of $f / f_{\text {force }}=1$. In addition, weaker spikes can also be seen at the second and third harmonics, $f / f_{\text {force }}=2$ and 3 , respectively. Narrow notch filters were added at $f / f_{\text {force }}=14.8$ and 29.6 to filter out nonphysical spikes originating from the anemometer. The influence of the second and third harmonics, as well as the subharmonic, was studied through a modal decomposition with the use of a bandpass filter around each relevant frequency. This exercise revealed that the fundamental forcing frequency gave rise to a significant portion of the synthetic mode energy and thus could be considered to be primarily responsible for the modal shape seen in Fig. 7a.

The frequency spectrogram was converted to a wavenumber spectrogram using the local mean velocity and Taylor's frozen field hypothesis and is shown in Fig. 9b. An increase in the turbulence, related to the small-scale structures, can be seen between $y / \delta=0.1$ and 0.5 and for $\lambda_{x} / \delta<3$. Above $\lambda_{x} / \delta=3$, sharp lines, related to the forcing frequency and the harmonics, dominate the spectra. The wavenumber spectrogram was used to determine a suitable cutoff criterion to separate the small- and large-scales following [25]. A cutoff criterion of $\lambda_{x} / \delta=3$ (in inner units, $\lambda_{x}^{+} \sim 22,800$ ), shown in Fig. $9 \mathrm{~b}$ as a vertical dashed line, was chosen to exclude the effect of the second and third harmonics and to best create a separation between the synthetic scale and the small-scales of turbulence. All scales above this cutoff criterion correspond to the large-scale component that includes the forcing frequencies and its significant harmonics, and all scales less than this cutoff criterion to the smallscale activity. The forcing wavelength, that is, the spacing between the external vortical structures, is approximately $8.6 \delta$.

Note that the chosen cutoff value is larger than at typical value of $\lambda_{x} / \delta \sim 1$ used to study the turbulence modulation in the canonical boundary layers (see, for instance, $[5,6]$ ). In this experiment, the boundary layer is modified by the external forcing, and structures larger than $\lambda_{x} / \delta>1$ become more energetic, as seen in Fig. 9b, thus requiring a larger cutoff value to properly separate the small-scale and 

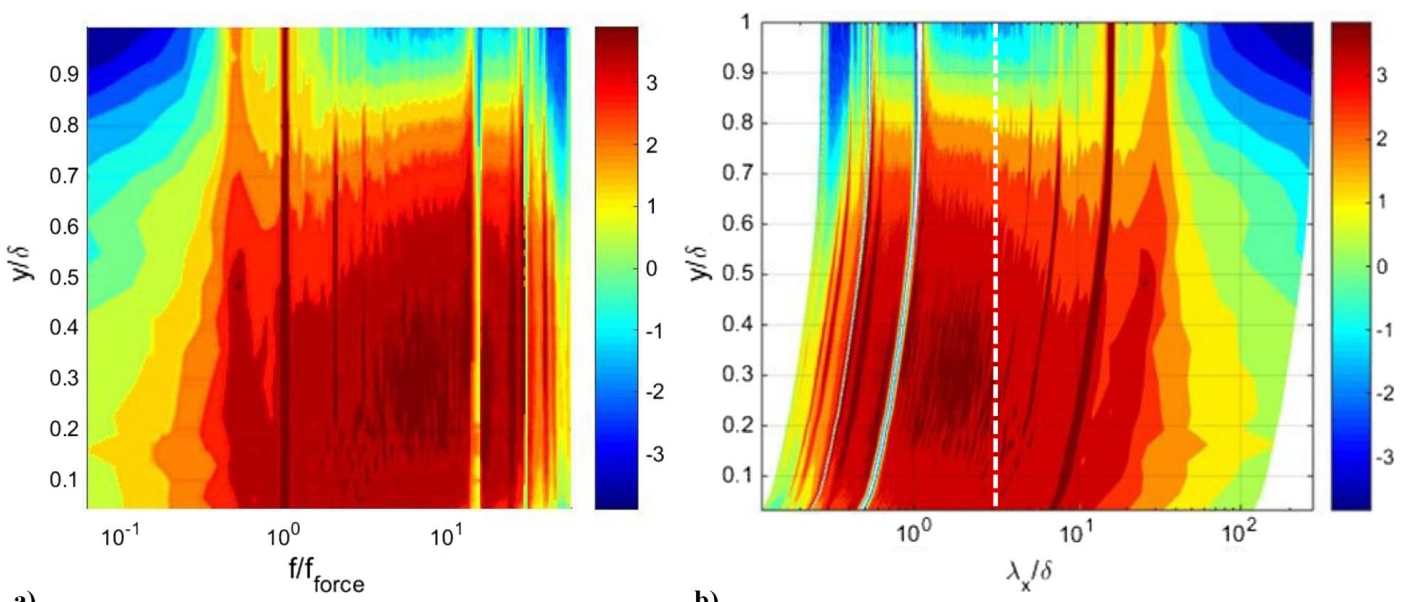

a)

Fig. 9 Logarithm of a premultiplied streamwise energy spectra (in arbitrary units) as a function of frequency, normalized by the forcing frequency, $f_{\text {force }}$ (a), and wavenumber (b). A cutoff criterion of $\lambda_{x} / \delta=3$, separating small- and large-scale structures, is indicated by a vertical dashed line in (b).

the large-scale forcing-related structures. An additional justification of the chosen cutoff criterion will be provided later in this paper.

The phase modulation and the related amplitude amplification of the turbulence were analyzed using the correlation coefficients following the works of Hutchins and Marusic [5], Mathis et al. [6] , and Duvvuri and McKeon [25]. Following [5,6], the mean-removed original temporal velocity signal, $u(y, t)$, at every wall-normal location was split into a large-scale, $u_{L}$, and small-scale, $u_{S}$, components using $\lambda_{x} / \delta=3$ as the wavenumber cutoff criterion via a finite impulse response (FIR) digital filter with a stopband attenuation of $-60 \mathrm{~dB}$. The concept of modulation of the smallscales by the large-scales in the flow is described extensively in [6], and the degree of modulation is represented by $R$, a single-point modulation correlation coefficient,

$$
R(y)=\frac{\overline{u_{L} E_{L}\left(u_{S}\right)}}{\sqrt{\overline{u_{L}^{2}} \sqrt{\overline{E_{L}\left(u_{S}\right)^{2}}}}}
$$

$E\left(u_{S}\right)$ represents the mean-removed envelope of the small-scale signal obtained using the Hilbert transform, as described in [6]. This envelope is filtered using the same low-pass filter to obtain the largescale component of the envelope itself, and in the expression for $R$, Eq. (4), this term is represented as $E_{L}\left(u_{S}\right)$.

The $R$-coefficient was computed throughout the boundary layer and is shown in Fig. 10. For comparison, the $R$-coefficient for a canonical boundary layer from [6] is also shown in Fig. 10. In comparing these plots, a few things are immediately noticeable. First, the shape of the correlation coefficient in the current work is similar to the plot for the canonical boundary layer. Second, there is a zero-crossing in the correlation coefficient with a positive correlation below $y / \delta \sim 0.4$ and a negative correlation above it. This indicates that the large-scales created by the external traveling structures are clearly having a modulating effect on the envelope of the small-scales of turbulence, in the same manner as naturally present large-scale structures modulate the scale-scale structures in an unperturbed boundary layer. Consistent with earlier observation about the presence of the critical layer, Fig. 10 also suggests the presence of a critical layer-type phenomenon, occurring around $y / \delta=0.4$. Below this location the small-scales are in phase with the large-scales, and above the location they are out of phase. When interpreted in the context of phase relationships, the plot also indicates that farther from the wall above the critical layer the small-scales lag behind the large-scales.

To demonstrate the validity of choosing $\lambda_{x} / \delta=3$ as the cutoff criterion, the amplitude modulation coefficient as a function of wallnormal distance, $R(y)$, was computed over a wide range of cutoff criteria between $\lambda_{x} / \delta=1$ and $\lambda_{x} / \delta=10$. Results (not shown here, see [28]) indicate that choosing a cutoff wavelength any larger than $5 \delta$ not only falsely characterizes stronger harmonics as "small"-scales,

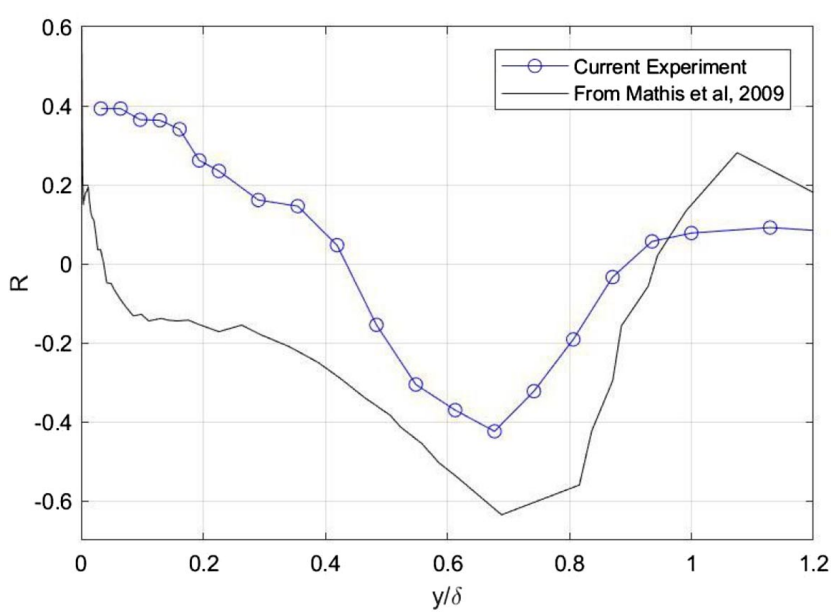

Fig. 10 Amplitude modulation correlation coefficient, $R$, for the forced boundary layer (open circles) and a modulation correlation coefficient between the large-scale component and the filtered envelope of the smallscale component for a canonical boundary layer, adapted from Mathis et al. [6] (solid line).

but also lead to a lack of convergence in the correlation coefficient closer to the wall. On the other hand, as can be seen in Fig. 9b, the peak associated with the broad band turbulent structure is located around $\lambda_{x} / \delta=2$ at $y / \delta=0.3$. Thus, choosing a cutoff wavelength any smaller than about $3 \delta$ for the studied boundary layer is not appropriate either, as in that case some of the actual small-scale turbulence would get falsely categorized as "synthetic" forcingrelated scales, and the modulation coefficient would underestimate the correlation. Thus, at least for this experiment, a cutoff wavelength between $3 \delta$ and $5 \delta$ seems the most appropriate, and so the selected cutoff criterion $\lambda_{x} / \delta=3$ was used in this analysis. For a similarly forced boundary layer in [25], a cutoff of $\lambda_{x} / \delta=5$ was chosen, using similar argumentation.

The boundary layer is next studied in a similar fashion to the internally forced case [25], to quantify the phase-organizing effect of the synthetic large-scale on the small-scales. As mentioned before, the work of Duvvuri and McKeon $[25,51]$ most closely resembles the current work, as in both cases the turbulent boundary layer was excited at a specific forcing frequency, and a synthetic large-scale was created in the hopes of studying its organizing effect on the other scales.

The importance of the critical layer is still a very active topic of research, and generalizations about critical layers for a canonical turbulent boundary layer cannot necessarily be applied to the noncanonical boundary layer in this experiment. The wall-normal location of the critical layer corresponded by definition to the location 
where the convection velocity of the internally created large-scale disturbance equals the local mean. In the internally forced boundary layer in $[25,51]$, it also corresponded to the location where the synthetic velocity mode energy had a peak. For the current experiment, the peak of the modal velocity energy occurred at $y / \delta=0.4$ as seen in Fig. $7 \mathrm{~b}$, where the local convection velocity is roughly $0.8 U_{0}$. The convection velocity of the large-scale disturbance was roughly $0.6 U_{0}$, and the wall-normal location where the convection velocity of the disturbance equaled the local mean velocity was approximately $y / \delta=0.2$. For this reason, the region $0.2<y / \delta<0.4$ was identified as a band representing the estimated region containing the true critical layer location; this region coincides with the region of the largest turbulence increase in Figs. $7 \mathrm{c}$ and 9. Additional investigation will be required to understand the potential importance - or lack thereof - of critical layer physics in this flow.

In [25], the authors developed a modified way to study the direct interaction of the synthetic large-scale on the small-scales of turbulence. Knowing that the small-scales are governed by the rules of triadic interactions between scales due to the quadratic nonlinearity in the Navier-Stokes equation, they pointed out that the synthetic large-scale has an associated wave number $k_{\mathrm{SLS}}$, and small-scales with wavenumbers $k_{n}$ and $k_{m}$, for example, interact with the synthetic scale in a triadically consistent manner such that $k_{\mathrm{SLS}}=k_{n}-k_{m}$. They showed that the square of the decomposed phase-locked averaged and mean-removed small-scale velocity signal, $\tilde{\varepsilon}=u_{S}^{2}$, represents the average triadic envelope of all smallscale wavenumbers $\left(k_{m}, k_{n}\right)$ that are directly coupled to the synthetic large-scale. Finally, the authors defined a new modulation correlation coefficient, $\Psi$, as the correlation coefficient between the modal component of the velocity due to forcing and the small-scale component of the signal, both obtained through phase-averaging with respect to the forcing signal,

$$
\Psi(y)=\frac{\langle\tilde{u}(y, \phi) \tilde{\varepsilon}(y, \phi)\rangle_{\phi}}{\sqrt{\left\langle\tilde{u}(y, \phi)^{2}\right\rangle_{\phi}} \sqrt{\left\langle\tilde{\varepsilon}(y, \phi)^{2}\right\rangle_{\phi}}}
$$

where the angle brackets define the phase averaging. To separate small-scales, the same cutoff criterion $\lambda_{x} / \delta=3$ was used in this work.

Alternatively, instead of $\tilde{\varepsilon}$, the overall mean-removed turbulence levels, $\Delta u_{\mathrm{rms}}^{\prime}(y, \phi)$, can be used to define a modulation correlation coefficient between the modal component $\tilde{u}(y, \varphi)$ and all other scales, as proposed in [52],

$$
\Phi(y)=\frac{\left\langle\tilde{u}(y, \phi) \Delta u_{\mathrm{rms}}^{\prime}(y, \phi)\right\rangle_{\phi}}{\sqrt{\left\langle\tilde{u}(y, \phi)^{2}\right\rangle_{\phi}} \sqrt{\left\langle\Delta u_{\mathrm{rms}}^{\prime}(y, \phi)^{2}\right\rangle_{\phi}}}
$$

The $R$-coefficient in Eq. (4) indicates the phase between the largescale, which includes the externally forced modal component and the small-scales. The $\Psi$-coefficient in Eq. (5) and the $\Phi$-coefficient in Eq. (6) indicate the phase between the modal velocity component and all the small-scales (or all scales, in case of $\Phi$-coefficient) that are in direct triadic coupling with the modal component and thus indicates a direct influence of the modal component on these scales.

Both $\Psi$ - and $\Phi$-coefficients were computed for the externally forced turbulent boundary layer in this work and are shown in Fig. 11, along with the $\Psi$-coefficient for the internally forced boundary layer from [25]. For the experiment in [25], the location of the critical layer was at $y / \delta=0.07$, labeled as a vertical dashed line in Fig. 11, and there was a strong positive correlation below the critical layer, and a strong negative correlation above the critical layer. The authors concluded that the synthetic large-scale was directly driving the small-scales to be in and out of phase with it below and above the critical layer. A similar, although not as pronounced, trend can be seen in $\Psi$-coefficient in the current experiment; see Fig. 11, where the critical layer band, estimated to be between $y / \delta$ of 0.25 and 0.4 , is indicated as a gray region. Below the critical layer band at $y / \delta<0.2$, there is a strong positive $(\Psi \sim 0.9)$ correlation, and above this layer, at $y / \delta>0.5$ there is a negative $(\Psi \sim-0.4)$ correlation, indicating that

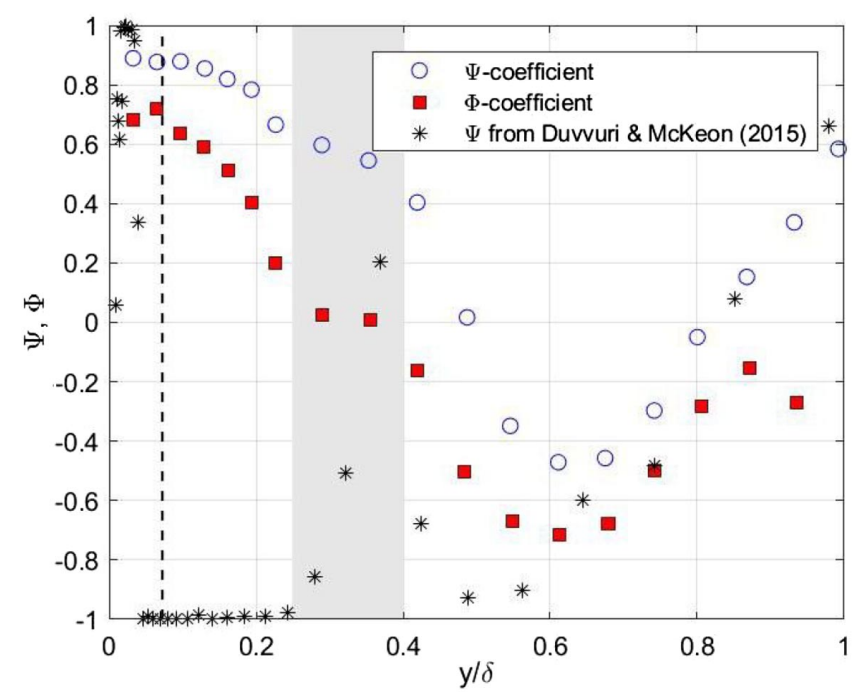

Fig. 11 Modulation correlation coefficients $\Psi$, defined in Eq. (5) (open circles), and $\Phi$, defined in Eq. (6) (filled squares). Gray region indicates the critical layer band for the current work. For comparison, the modulation correlation coefficient $\Psi$ between synthetic scale and smallscale turbulence from Duvvuri and McKeon [25] (black asterisks) is shown for comparison, and a vertical dashed line indicates the location of the critical layer from [25].

the synthetic large-scale introduced by the shear layer drives the small-scales of turbulence to be in and out of phase with it with respect to the critical location. Above this critical layer, the correlation coefficient quickly approaches the intermittent turbulent/ nonturbulent interface of the flow; it is suspected that for this reason the anticorrelation above the critical location is not as strong as that seen in [25] and does not have a large wall-normal extent. The $\Phi$ coefficient shows very similar trends, with large positive values below the critical band and large negative values above it. Zero crossing in $\Phi$-coefficient is closer to the wall, around $y / \delta=0.3$; the similar trend was observed in [52] as well.

It is worth noting that for the present studies, there is no sharp change in $\Psi$-coefficient across the critical layer, as it was observed in $\Psi$-coefficient from [25], where it quickly changed from 1 to -1 across the critical layer. One possible explanation is that the shearlayer induced disturbance cannot be assumed to be 2-D, unlike in [25]. Also, the ratio of test-section width to boundary-layer thickness is relatively small, about 5.2. Thus, the lack of the sharp changes in both $\Psi$ - and $\Phi$-coefficients across the critical layer band likely indicates that a spanwise-varying forcing is more closely aligned with the real large-scale variation than the previous, essentially spanwise constant, dynamic roughness studies in [25]. Additional studies are needed to further investigate this observation.

\section{Conclusions}

In an attempt to explore what appeared to be turbulence "bursts" or amplification in an aero-optical study of a forced shear layer, a Mach 0.6 turbulent boundary layer was externally forced using a regularized shear layer, which created a concomitant unsteady pressure field that provided a disturbance for the turbulent boundary layer on the upper wall of the tunnel. The turbulence amplification and the related phase modulation inside the boundary layer, caused by the periodic external forcing by the shear layer, were observed and studied using hotwire anemometry. A phase-locking algorithm was implemented to align the hot-wire results to the deterministic passing of the coherent vortical structures in the shear layer. Regions of amplified turbulence inside the boundary layer were observed between $y / \delta=0.2$ and 0.4 . These regions were found to be slightly ahead in phase of the external forcing shear-layer structures. The convective speed of these regions was found to be in the range $0.6 U_{0}-0.8 U_{0}$, where $U_{0}$ is the freestream speed outside the boundary, close to the convective speed of the shear-layer structures 
$\left(0.56 U_{0}\right)$. The matched speeds suggest the existence of critical layer inside the boundary layer, which may be correlated with the turbulence amplification regions. Various modulation correlation coefficients were computed, and they also indicated the existence of the critical layer near $y / \delta=0.4$. The phase-locked results were compared with the results of the internally forced boundary layer and were found to be in general agreement with them.

Although this study has investigated external forcing of a compressible boundary layer, strong similarities were found with earlier work on quasi-steady modulation based on the passage of energetic large-scale structures, naturally occurring in turbulent boundary layers. In addition, the research presented in this paper demonstrates that the amplitude modulation phenomenon, originally observed in low-speed boundary layers, also occurs in the highMach-number subsonic regime, where commercial aircraft typically operate, and warrants the need to continue studying this topic. The current research adds to the growing database of flow studies that have documented similar modulation correlation coefficient curves representing the organization of structures within the boundary layer.

\section{Acknowledgments}

This effort was funded in part by the High Energy Laser-Joint Technology Office (HEL-JTO) and administered through the Air Force Office for Scientific Research (AFOSR) under Grant Number FA9550-13-1-0001. The U.S. Government is authorized to reproduce and distribute reprints for governmental purposes notwithstanding any copyright notation thereon.

\section{References}

[1] Kline, S. J., Reynolds, W. C., Schraub, F. A., and Runstadler, P. W., "The Structure of Turbulent Boundary Layers," Journal of Fluid Mechanics, Vol. 30, No. 4, 1967, pp. 741-773.

[2] Rao, K., Narasimha, R., and Narayanan, M., "The 'Bursting' Phenomenon in a Turbulent Boundary Layer," Journal of Fluid Mechanics, Vol. 48, No. 2, 1971, pp. 339-352. doi:10.1017/S0022112071001605

[3] Bandyopadhyay, P. R., and Hussain, A. K. M. F., "The Coupling Between Scales in Shear Flows," Physics of Fluids, Vol. 27, No. 9, 1984, pp. 2221-2228. doi:10.1063/1.864901

[4] Brown, G. L., and Thomas, A. S. W., "Large Structure in a Turbulent Boundary Layer," Physics of Fluids, Vol. 20, No. 10, 1977, p. S243. doi:10.1063/1.861737

[5] Hutchins, N., and Marusic, I., "Large-Scale Influences in Near-Wall Turbulence," Philosophical Transactions of the Royal Society A, Vol. 365, No. 1852, 2007, pp. 647-664. doi:10.1098/rsta.2006.1942

[6] Mathis, R., Hutchins, N., and Marusic, I., "Large-Scale Amplitude Modulation of the Small-Scale Structures in Turbulent Boundary Layers," Journal of Fluid Mechanics, Vol. 628, June 2009, pp. 311-336. doi:10.1017/S0022112009006946

[7] Mathis, R., Monty, J. P., Hutchins, N., and Marusic, I., "Comparison of Large-Scale Amplitude Modulation in Turbulent Boundary Layers, Pipes, and Channel Flows," Physics of Fluids, Vol. 21, No. 11, 2009, Paper 111703. doi:10.1063/1.3267726

[8] Mathis, R., Hutchins, N., and Marusic, I., "A Predictive Inner-Outer Model for Streamwise Turbulence Statistics in Wall-Bounded Flows," Journal of Fluid Mechanics, Vol. 681, Aug. 2011, pp. 537-566. doi:10.1017/jfm.2011.216

[9] Townsend, A. A., The Structure of Turbulent Shear Flow, 2nd ed., Cambridge Univ. Press, Cambridge, U.K., 1976, pp. 105-118.

[10] Mathis, R., Marusic, I., Chernyshenko, S. I., and Hutchins, N., "Estimating Wall-Shear-Stress Fluctuations Given an Outer Region Input," Journal of Fluid Mechanics, Vol. 715, Jan. 2013, pp. 163-180. doi:10.1017/jfm.2012.508

[11] Zhang, C., and Chernyshenko, S. I., "Quasisteady Quasihomogeneous Description of the Scale Interactions in Near-Wall Turbulence," Physical Review Fluids, Vol. 1, No. 1, 2016, Paper 014401. doi:10.1103/PhysRevFluids.1.014401

[12] Kim, K. C., and Adrian, R. J., "Very Large-Scale Motion in the Outer Layer," Physics of Fluids, Vol. 11, No. 2, 1999, pp. 417-422. doi: $10.1063 / 1.869889$
[13] Jacobi, I., and McKeon, B. J., "Phase Relationships Between Large and Small Scales in the Turbulent Boundary Layer," Experiments in Fluids, Vol. 54, No. 3, 2013, p. 1481. doi:10.1007/s00348-013-1481-y

[14] Karlsson, S. K. F., "An Unsteady Turbulent Boundary Layer," Journal of Fluid Mechanics, Vol. 5, No. 4, 1959, pp. 622-636. doi:10.1017/S0022112059000428

[15] Patel, M. H., "On Turbulent Boundary Layers in Oscillatory Flow," Proceedings of the Royal Society of London Series A, Vol. 353, No. 1672, 1977, pp. 121-144. doi:10.1098/rspa.1977.0025

[16] Simpson, R. L., Shivaprasad, B. G., and Chew, Y.-T., "The Structure of a Separating Turbulent Boundary Layer. Part 4. Effects of Periodic FreeStream Unsteadiness," Journal of Fluid Mechanics, Vol. 127, Feb. 1983, pp. 219-261. doi: $10.1017 / \mathrm{S} 0022112083002700$

[17] Brereton, G. J., Reynolds, W. C., and Jayaraman, R., "Response of a Turbulent Boundary Layer to Sinusoidal Free- Stream Unsteadiness," Journal of Fluid Mechanics, Vol. 221, Dec. 1990, pp. 131-159. doi:10.1017/S0022112090003512

[18] Ramaprian, B. R., and Tu, S. W., "Fully Developed Periodic Turbulent Pipe Flow. Part 2. The Detailed Structure of the Flow," Journal of Fluid Mechanics, Vol. 137, Dec. 1983, pp. 59-81. doi:10.1017/S0022112083002293

[19] Brereton, G. J., and Mankbadi, R. R., "Review of Recent Advances in the Study of Unsteady Turbulent Internal Flows," Applied Mechanical Reviews, Vol. 48, No. 4, 1995, pp. 189-212. doi: $10.1115 / 1.3005100$

[20] McKeon, B. J., and Sharma, A. S., "A Critical-Layer Framework for Turbulent Pipe Flow,” Journal of Fluid Mechanics, Vol. 658, Sept. 2010, pp. 336-382. doi:10.1017/S002211201000176X

[21] McKeon, B. J., "The Engine Behind (Wall) Turbulence: Perspectives on Scale Interactions," Journal of Fluid Mechanics, Vol. 817, April 2017, p. P1. doi: $10.1017 / \mathrm{jfm} .2017 .115$

[22] Sharma, A. S., and McKeon, B. J., "On Coherent Structure in Wall Turbulence," Journal of Fluid Mechanics, Vol. 728, Aug. 2013, pp. 196-238. doi:10.1017/jfm.2013.286

[23] Jacobi, I., and McKeon, B. J., "Dynamic Roughness Perturbation of a Turbulent Boundary Layer," Journal of Fluid Mechanics, Vol. 688, Dec. 2011, pp. 258-296.

[24] McKeon, B. J., Sharma, A. S., and Jacobi, I., "Experimental Manipulation of Wall Turbulence: A Systems Approach," Physics of Fluids, Vol. 25, No. 3, 2013, Paper 031301. doi:10.1063/1.4793444

[25] Duvvuri, S., and McKeon, B. J., "Triadic Scale Interactions in a Turbulent Boundary Layer," Journal of Fluid Mechanics, Vol. 767, March 2015, p. R4. doi:10.1017/jfm.2015.79

[26] Duffin, D. A., "Feed-Forward Adaptive-Optic Correction of A WeaklyCompressible High-Subsonic Shear Layer," Ph.D. Dissertation, Univ. of Notre Dame, Notre Dame, IN, 2009.

[27] Dogan, E., Hanson, R., and Ganapathisubramani, B., "Interactions of Large-Scale Free-Stream Turbulence with Turbulent Boundary Layers," Journal of Fluid Mechanics, Vol. 802, Sept. 2016, pp. 79-107. doi: $10.1017 / \mathrm{jfm} .2016 .435$

[28] Ranade, P. M., "Turbulence Amplitude Modulation in an Externally Forced, High Reynolds Number Boundary Layer," Ph.D. Dissertation, Univ. of Notre Dame, Notre Dame, IN, 2016.

[29] Oster, D., and Wygnanski, I. J., "The Forced Mixing Layer Between Parallel Streams," Journal of Fluid Mechanics, Vol. 123, Oct. 1982, pp. $91-130$. doi: $10.1017 / \mathrm{S} 0022112082002973$

[30] Rennie, M., Siegenthaler, J., and Jumper, E., "Forcing of a TwoDimensional, Weakly-Compressible Subsonic Free Shear Layer," AIAA Paper 2006-561, 2006.

[31] Hutchins, N., Nickels, T., Marusic, I., and Chong, M., "Hot-Wire Spatial Resolution Issues in Wall Bounded Turbulence," Journal of Fluid Mechanics, Vol. 635, Sept. 2009, pp. 103-136. doi:10.1017/S0022112009007721

[32] Cukurel, B., Acarer, S., and Arts, T., "A Novel Perspective to HighSpeed Cross-Hot-Wire Calibration Methodology," Experiments in Fluids, Vol. 53, No. 4, 2012, pp. 1073-1085. doi:10.1007/s00348-012-1344-y

[33] Nagib, H. M., Chauhan, K. A., and Monkewitz, P. A., "Approach to an Asymptotic State for Zero Pressure Gradient Turbulent Boundary Layers," Philosophical Transactions of the Royal Society A, Vol. 365, 
No. 1852,2007 , pp. $755-770$.

doi: $10.1098 /$ rsta.2006.1948

[34] Boiko, A. V., and Kornilov, V. I., "Effect of Periodic Blowing/Suction Through Sequentially Located Annular Slots on the Turbulent Boundary Layer on a Body of Revolution," Thermophysics and Aeromechanics, Vol. 15, No. 1, 2008, pp. 11-27. doi:10.1134/S0869864308010022

[35] Nagib, H. M., Chauhan, K. A., and Monkewitz, P. A., "Approach to an Asymptotic State for Zero Pressure Gradient Turbulent Boundary Layers," Philosophical Transactions of the Royal Society A, Vol. 365, No. 1852, 2007, pp. 755-770. doi:10.1098/rsta.2006.1948

[36] Nagib, H. M., and Chauhan, K. A., "Variations of von Kármán Coefficient in Canonical Flows," Physics of Fluids, Vol. 20, No. 10, 2008, Paper 101518 doi:10.1063/1.3006423

[37] Papamoschou, D., and Roshko, A., "The Compressible Turbulent Shear Layer: An Experimental Study," Journal of Fluid Mechanics, Vol. 197, Dec. 1988 , pp. $453-477$ doi:10.1017/S0022112088003325

[38] Barre, S., Dupont, P., and Dussauge, J. P., "Estimates of Convection Velocity of Large Turbulent Structures in Supersonic Mixing Layers," Aerospace Science and Technology, Vol. 1, No. 5, 1997, pp. 355-366. doi:10.1016/S1270-9638(97)90056-0

[39] Agostini, L., Leschziner, M., Poggie, J., Bisek, N. J., and Gaitonde, D., "Multi-Scale Interactions in a Compressible Boundary Layer," Journal of Turbulence, Vol. 18, No. 8, 2017, pp. 760-780. doi:10.1080/14685248.2017.1328108

[40] Hussain, A. K. M. F., and Reynolds, W. C., "The Mechanics of an Organized Wave in Turbulent Shear Flow," Journal of Fluid Mechanics, Vol. 41, No. 2, 1970, pp. 241-258. doi:10.1017/S0022112070000605

[41] Smits, A. J., McKeon, B. J., and Marusic, I., "High-Reynolds Number Wall Turbulence," Annual Review of Fluid Mechanics, Vol. 43, No. 1, 2011, pp. 353-375. doi:10.1146/annurev-fluid-122109-160753

[42] Klewicki, J., Fife, P., Wei, T., and Mcmurtry, P., "A Physical Model of the Turbulent Boundary Layer Consonant with Mean Momentum Balance Structure," Philosophical Transactions of the Royal Society of London Series A, Vol. 365, No. 1852, 2007, pp. 823-840. doi:10.1098/rsta.2006.1944

[43] Reynolds, W. C., and Hussain, A. K. M. F., "The Mechanics of an Organized Wave in Turbulent Shear Flow. Part 3. Theoretical Models and Comparisons with Experiments," Journal of Fluid Mechanics,
Vol. 54, No. 2, 1972, pp. 263-288. doi:10.1017/S0022112072000679

[44] Baars, W. J., Hutchins, N., and Marusic, I., "Reynolds Number Trend of Hierarchies and Scale Interactions in Turbulent Boundary Layers," Philosophical Transactions of the Royal Society of London Series A, Vol. 375, No. 2089, 2017, Paper 20160077. doi:10.1098/rsta.2016.0077

[45] Baars, W. J., Talluru, K. M., Hutchins, N., and Marusic, I., "Wavelet Analysis of Wall Turbulence to Study Large-Scale Modulation of Small Scales," Experiments in Fluids, Vol. 56, No. 10, 2015, p. 188. doi:10.1007/s00348-015-2058-8

[46] Sutton, G. W., "Effect of Turbulent Fluctuations in an Optically Active Fluid Medium," AIAA Journal, Vol. 7, No. 9, 1969, pp. 1737-1743. doi: $10.2514 / 3.5384$

[47] Gordeyev, S., Smith, A. E., Cress, J. A., and Jumper, E. J., "Experimental Studies of Aero-Optical Properties of Subsonic Turbulent Boundary Layers," Journal of Fluid Mechanics, Vol. 740, Feb. 2014, pp. 214-253. doi:10.1017/jfm.2013.658

[48] Gordeyev, S., and Juliano, T. J., "Optical Characterization of NozzleWall Mach-6 Boundary Layers," AIAA Paper 2016-1586, 2016.

[49] Gordeyev, S., Cress, J. A., Smith, A., and Jumper, E. J., "Aero-Optical Measurements in a Subsonic, Turbulent Boundary Layer with NonAdiabatic Walls," Physics of Fluids, Vol. 27, No. 4, 2015, Paper 045110. doi:10.1063/1.4919331

[50] Kemnetz, M., Gordeyev, S., Ranade, P., and Jumper, E. J., "Optical Investigation of Turbulence Modulation in an Externally Forced, High Reynolds Number Boundary Layer," Proceedings of 10th International Symposium on Turbulence and Shear Flow Phenomena (TSFP-10), Vol. 2, Chicago, July 2017.

[51] Duvvuri, S., and McKeon, B. J., "Small-Scale Phase Organization Through Large-Scale Inputs in a Turbulent Boundary Layer," Proceedings of 9th International Symposium on Turbulence and Shear Flow Phenomena (TSFP-9), Vol. 1, Melbourne, Australia, 2015.

[52] Duvvuri, S., and McKeon, B., "Phase Relations in a Forced Turbulent Boundary Layer: Implications for Modelling of High Reynolds Number Wall Turbulence," Philosophical Transactions. Series A, Mathematical, Physical, and Engineering Sciences, Vol. 375, No. 2089, 2017, Paper 20160080 .

L. Ukeiley

Associate Editor 